\title{
Modelling cell cycle synchronisation in networks of coupled radial glial cells
}

\author{
Duncan S Barrack ${ }^{\mathrm{a}, \mathrm{b}, 1}$, Rüdiger Thul ${ }^{\mathrm{b}}$ and Markus R Owen ${ }^{\mathrm{b}}$ \\ ${ }^{a}$ Horizon Digital Economy Research Institute, University of Nottingham, Nottingham, UK. \\ ${ }^{\mathrm{b}}$ School of Mathematical Sciences and Centre for Mathematical Medicine and Biology, \\ University of Nottingham, Nottingham, UK.
}

\begin{abstract}
Radial glial cells play a crucial role in the embryonic mammalian brain. Their proliferation is thought to be controlled, in part, by ATP mediated calcium signals. It has been hypothesised that these signals act to locally synchronise cell cycles, so that clusters of cells proliferate together, shedding daughter cells in uniform sheets. In this paper we investigate this cell cycle synchronisation by taking an ordinary differential equation model that couples the dynamics of intracellular calcium and the cell cycle and extend it to populations of cells coupled via extracellular ATP signals. Through bifurcation analysis we show that although ATP mediated calcium release can lead to cell cycle synchronisation, a number of other asynchronous oscillatory solutions including torus solutions dominate the parameter space and cell cycle synchronisation is far from guaranteed. Despite this, numerical results indicate that the transient and not the asymptotic behaviour of the system is important in accounting for cell cycle synchronisation. In particular, quiescent cells can be entrained on to the cell cycle via ATP mediated calcium signals initiated by a driving cell and crucially will cycle in near synchrony with the driving cell for the duration of neurogenesis. This behaviour is highly sensitive to the timing of ATP release, with release at the $G_{1} / S$ phase transition of the cell cycle far more likely to lead to near synchrony than release during mid $G_{1}$ phase. This result, which suggests that ATP release timing is critical to radial glia cell cycle synchronisation may help to understand normal and pathological brain development.
\end{abstract}

Keywords: cell cycle; calcium dynamics; radial glial cells; cell cycle synchronisation; bifurcation analysis.

\footnotetext{
${ }^{1}$ Corresponding author at: Horizon Digital Economy Research Institute, University of Nottingham, Nottingham, NG7 2TU, UK. Tel.: +44 (0)115 8232554. E-mail address: duncan.barrack@nottingham.ac.uk.
} 


\section{Introduction}

Radial glial cells give rise to the vast majority of neurons in the neocortex (Noctor et al., 2001). Only present for a brief period during embryonic development when they proliferate, all radial glial cells eventually differentiate into neurons. It has been demonstrated that radial glia release adenosine tri-phosphate (ATP) (Weissman et al., 2004) via hemichannels ( $\mathrm{Li}$ et al., 1996). The question of exactly when during the cell cycle hemichannels form has not been definitively answered in the literature. The experiments of Weissman et al. (2004) suggest that they may form in, or near, S-phase. Bittman and LoTurco (1999) investigated hemichannel opening in populations of ventricular zone cells which included radial glia. They found that the point of the cell cycle which corresponds to hemichannel opening is highly variable; opening was observed for part of $\mathrm{G}_{1}$, as well as during the entirety of $\mathrm{G}_{1}$ and also for part of S-phase and was not necessarily consistent for every cell in the population.

ATP is one of the primary means by which radial glia communicate and influences proliferation. By inhibiting the normal function of ATP receptors for one hour Weissman et al. (2004) observed that the proportion of cells labelled with BrdU (indicating S-phase entry) was 54.7\% that of the case where ATP receptors were allowed to function as normal. Although the key downstream factor affecting proliferation was not identified in the study, in radial glia ATP leads, via a G-protein cascade, to inositol 1,4,5-trisphosphate $\left(\mathrm{IP}_{3}\right)$ mediated calcium release from the endoplasmic reticulum (ER) (Weissman et al., 2004). Importantly there is evidence that calcium influences cell cycle progression in many cell types (Berridge, 1995; Berridge et al., 2000). Calcium, via calcium/calmodulin dependent kinases, causes an increase in Cyclin D activity (Kahl and Means, 2003, 2004; Morris et al., 1998; Tombes et al., 1995; Rasmussen and Rasmussen, 1995), a key cell cycle protein crucial for $\mathrm{G}_{1}$ progression. In addition to the roll of ATP in cellular proliferation it has also been conjectured that the ATP mediated calcium signalling mechanism is responsible for synchronising the cell cycles of clusters of radial glia (Weissman et al., 2004). Release from a handful of cells may have the effect of synchronising the cell cycles within a cluster, or cohort, of cells as well as recruiting cells in $\mathrm{G}_{0}$ on to the cell cycle (see Figure 1). Synchronised radial glia lead to the birth of daughter cells from the cohort in an ordered, regular fashion. It has been speculated that the neurons which originate from the same synchronised cohort go on to reside in the same cortical layer (Weissman et al., 2004). Hence, radial glia synchronisation has implications for the architecture of the developing mammalian brain. 
In (Barrack et al., 2014), we introduced a model for coupled calcium-cell cycle dynamics in a single radial glial cell which is outlined in Appendix A. We showed that ATP mediated calcium release led to a modest increase in the frequency of the cell cycle. The modulation in frequency, in itself, could not explain the experimentally observed fluctuations in proliferation rates associated with ATP. However, our analysis revealed an area of multistability in which stable fixed points and limit cycle solutions coexist. This area allows for a proliferating radial glial cell to recruit an otherwise quiescent cell onto the cell cycle via an ATP mediated calcium signalling mechanism. This in turn could explain the changes in cellular proliferation which have been observed experimentally (Weissman et al., 2004).

In this paper we investigate whether, and under what conditions, an ATP mediated calcium signalling mechanism leads to cell cycle synchronisation of clusters of radial glia. Systems of cells coupled via extracellular ATP on one and two dimensional lattices are considered and the system dynamics are studied via bifurcation analysis and direct numerical simulations. We consider clusters in which all cells are initially cycling and, because our previous work suggests that quiescent cell recruitment is extremely important, we also investigate cell cycle synchrony under the scenario in which some cells within the cluster are initially dormant. Our results indicate that stable synchronous solutions are far from guaranteed. However, our model does permit trajectories where cells cycle in near synchrony for a period of time consistent with the period of neurogenesis in mammals. For our model, cells have a greater propensity to synchronise when ATP is released at the $\mathrm{G}_{1} / \mathrm{S}$ phase transition rather than during mid $\mathrm{G}_{1}$ phase of the cell cycle. As radial glial cell cycle synchronisation is crucial for the regular formation of the neocortex, these results may shed light on the causes of some neurodevelopment disorders which stem from irregular cortical formation.

This paper is structured as follows. In Section 2 we outline our model for ATP mediated calcium-cell cycle coupling in radial glia. In Section 3, we present our results before concluding with a discussion in Section 4.

\section{The model}

Our single cell model, introduced in (Barrack et al., 2014), is comprised of a cell cycle component based on the model of Obeyesekere et al. (1999) and an ATP mediated calcium release component based on the model of Bennett et al. (2005). The cell cycle model includes five dynamical variables including Cyclin D/Cdk4, Cy- 
a)

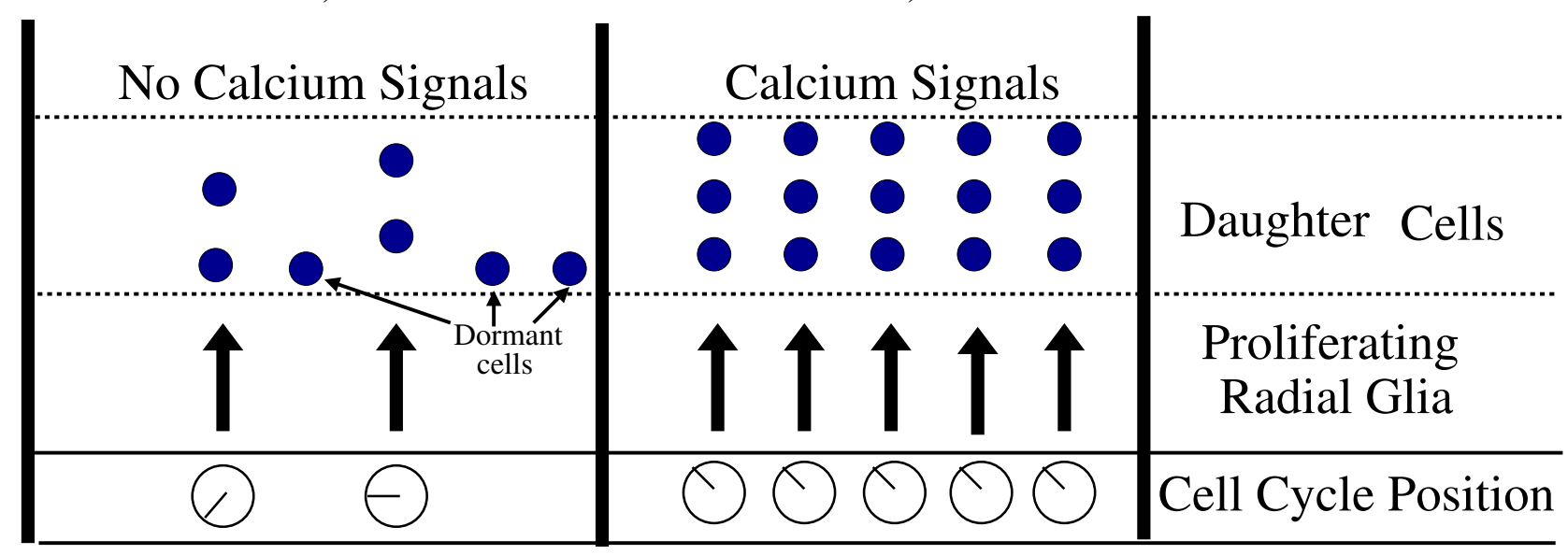

Figure 1: Schematic illustrating how ATP mediated calcium signals initiated by a small number of cycling cells may not only recruit otherwise quiescent cells on to the cell cycle but also synchronise the cell cycles of radial glial cells, so that clusters of cells cycle in unison. (a) No calcium present. Here two cells proliferate in an asynchronous manner (represented by the position of the clock hands) whilst the remainder remain dormant and do not cycle. (b) Dormant cells are recruited on to the cell cycle via ATP mediated calcium release from cycling cells which also has the effect of synchronising cell cycles, leading to the shedding of daughter cells in uniform sheets.

clin E/Cdk2, unphosphorylated retinoblastoma tumour suppressor protein (RB) and phosphorylated RB bound to the E2F transcription factor. These proteins drive a cell through $\mathrm{G}_{1}$ phase into $\mathrm{S}$ phase of the cell cycle. The model also includes a 'cell progression indicator' variable which indirectly represents the kinases, phosphatases and proteases responsible for driving the cell through the remainder of $\mathrm{S}, \mathrm{G}_{2}$ and $\mathrm{M}$ phases of the cell cycle. The ATP mediated calcium release model component accounts for calcium release from ER and includes variables for extra and intracellular ATP concentrations, proportion of external P2 $\mathrm{Y}_{1}$ receptors bound to ATP, G-protein activation and $\mathrm{IP}_{3}$ production. In our model, the coupling between the calcium dynamics and cell cycle dynamics is mediated via the Cyclin D production rate which we make an increasing function of calcium in the cell. Each cell is coupled to its nearest neighbours via extracellular ATP. This ensures that when cells are cycling an ATP signal is passed on to its nearest neighbours which regenerate the signal by releasing their own ATP. As it is unclear when exactly hemichannels form and ATP is released two model variants with different ATP release patterns are considered. In the first variant, which we refer to as the 'Cyclin D model variant', hemichannel opening and ATP release is dependent on Cyclin D and ATP release occurs during mid $\mathrm{G}_{1}$ phase of the cell cycle. In the second variant, which we refer to as the ' $R_{\mathrm{s}}$ model variant', ATP is released at the $\mathrm{G}_{1} / \mathrm{S}$ phase transition. Here, $R_{\mathrm{S}}$ denotes phosphorylated RB bound to the E2F transcription factor. The model equations and parameters are given in Appendix A. 


\section{Results}

In order to investigate the hypothesis of Weissman et al. (2004) that ATP mediated calcium signals act to synchronise the cell cycles of clusters of radial glia, first we study the dynamics of multicellular systems for both model variants via bifurcation analysis and direct numerical simulation. We begin by conducting bifurcation analysis on two cell systems using the Cyclin D synthesis rate $a_{\mathrm{d} 2}^{\prime}$ of the second cell as the control parameter (Figure 2). $a_{\mathrm{d} 2}^{\prime}$ plays a crucial role in our model as it affects the frequency of oscillation of the cell cycle and can control whether the second cell oscillates at all. Furthermore, in reality there will be small physiological differences between radial glial cells and the parameter $a_{\mathrm{d} 2}^{\prime}$ can be regarded as a proxy for these differences. For the Cyclin D dependent model variant (Figures 2a and 2c), the stability of a limit cycle solution branch is lost at a torus bifurcation point (Kuznetsov, 1998) (TB at $a_{\mathrm{d} 2}^{\prime} \approx 0.380$ ), where it gives rise to quasi-periodic torus solutions. At $a_{\mathrm{d} 2}^{\prime} \approx 0.377$ a different limit cycle solution branch with higher Cyclin D concentrations is created via a saddle node bifurcation $\left(\mathrm{FP} 1^{D}\right)$. Additional pairs of saddle node bifurcations (FP2 ${ }^{D}$ at $a_{\mathrm{d} 2}^{\prime} \approx 0.4086$ and $\mathrm{FP} 3^{D}$ at $a_{\mathrm{d} 2}^{\prime} \approx 0.411$ ) are created on this solution branch leading to the coexistence of two sets of stable limit cycle solutions for $0.4086<a_{\mathrm{d} 2}^{\prime}<0.411$. The high Cyclin D concentration limit cycle solution branch is destroyed via a saddle node bifurcation point $\left(\mathrm{FP} 4{ }^{D}\right)$ at $a_{\mathrm{d} 2}^{\prime} \approx 0.440$. The bifurcation diagram for the $R_{\mathrm{S}}$ model variant (Figures $2 \mathrm{~b}$ and $2 \mathrm{~d}$ ) is similar to the Cyclin D variant except that torus solutions are permitted for lower values of $a_{\mathrm{d} 2}^{\prime}(\approx 0.360)$ and the branch of limit cycle solutions which arises from a saddle node bifurcation point (FP1 ${ }^{R s}$ at $\left.a_{\mathrm{d} 2}^{\prime} \approx 0.403\right)$ and vanishes at $a_{\mathrm{d} 2}^{\prime} \approx 0.417$ (marked FP2 ${ }^{R s}$ ) does not contain an additional saddle node bifurcation. The reason why torus solutions arise for lower values of $a_{\mathrm{d} 2}^{\prime}$ is that, under the parameter regime of Figure 2, ATP release for the $R_{\mathrm{S}}$ dependent model variant is of a longer duration than that of the Cyclin D dependent ATP release model variant. Longer release leads to a greater increase in Cyclin D production in the quiescent cell which is required to lift it from a low amplitude limit cycle solution corresponding to quiescence onto a higher amplitude oscillatory solution corresponding to the cycling state.

Next, to illustrate the degree of cell cycle synchronisation for the limit cycle and torus solutions uncovered by the bifurcation analysis, we present the results of numerical simulations. Figures $3 \mathrm{a}$ and $3 \mathrm{~b}$ for $a_{\mathrm{d} 2}^{\prime}=0.35$ show an example of the limit cycle solution where the amplitude of oscillation of cell 1 is far higher than that of cell 2. Here the Cyclin D concentrations of the second cell are so low that it is regarded as still being in the quiescent state (Obeyesekere et al., 1999). For $a_{\mathrm{d} 2}^{\prime}=0.4$, where stable torus and stable limit cycle solutions co- 
(a)

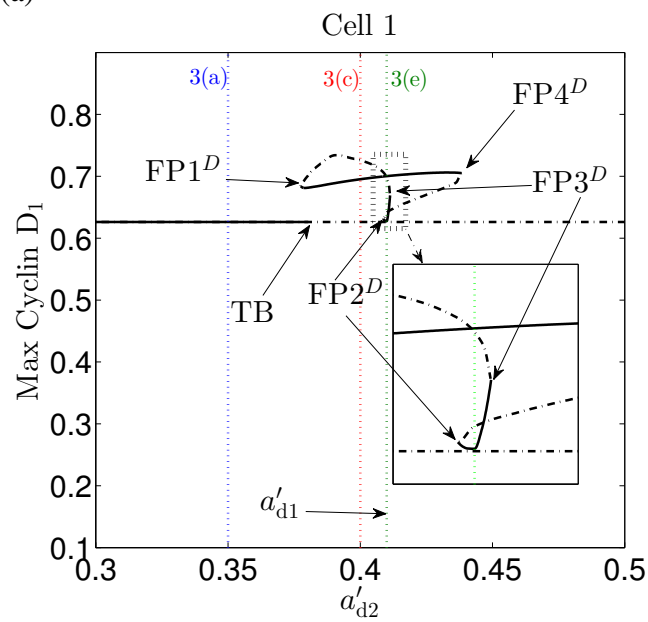

(c)

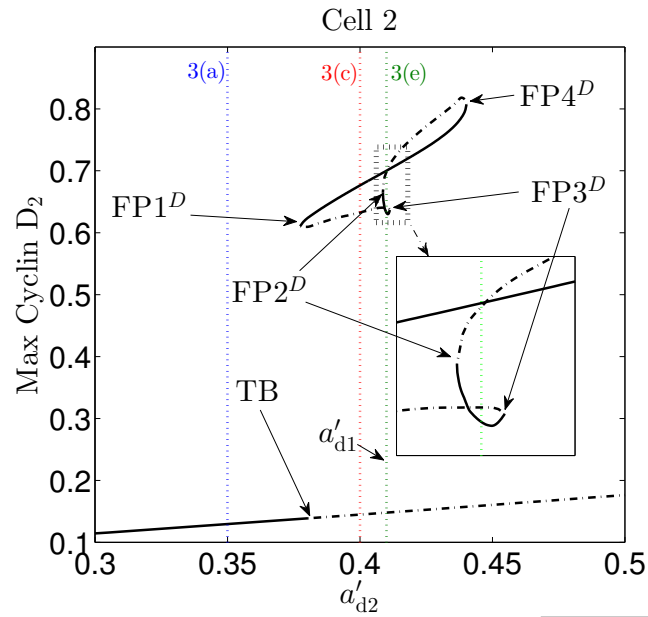

(b) $R_{\mathrm{S}}$ Dependent ATP Release

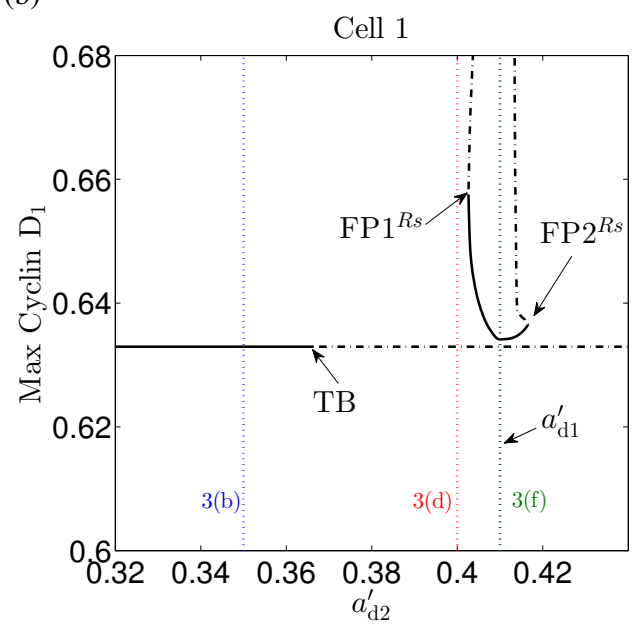

(d)

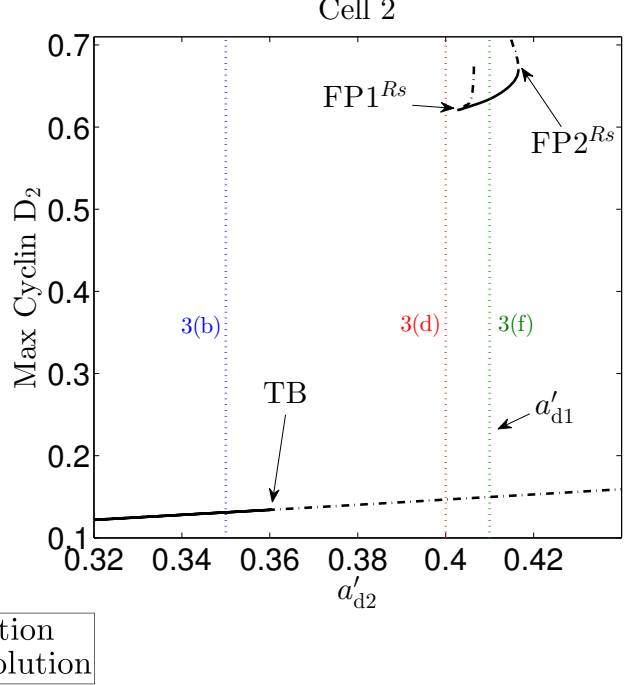

Figure 2: Bifurcation diagrams of system (A.1)-(A.13) for two radial glial cells coupled via the diffusion of extracellular ATP. The system permits several different limit cycle solutions as well as torus solutions. Plots show maximum Cyclin D concentration as a function of the intrinsic Cyclin D synthesis rate of the second cell $a_{\mathrm{d} 2}^{\prime}$. (a, c) correspond to the Cyclin D dependent ATP release model variant $\left(\left(i_{1}, i_{2}\right)=\left(D, D_{\mathrm{c}}\right)\right)$ where ATP is released around mid $\mathrm{G}_{1}$ phase of the cell cycle and $(\mathrm{b}, \mathrm{d})$ to the $R_{\mathrm{S}}$ dependent ATP release model variant $\left(\left(i_{1}, i_{2}\right)=\left(R_{\mathrm{Sc}}, R_{\mathrm{S}}\right)\right)$ where release occurs at the $\mathrm{G}_{1} / \mathrm{S}$ transition. $\mathrm{FP} 1^{D}, \mathrm{FP} 2^{D}, \mathrm{FP} 3^{D}, \mathrm{FP} 4^{D}, \mathrm{FP} 1^{R s}$ and FP2 ${ }^{R s}$ correspond to fold or saddle node bifurcation points, while TB corresponds to a torus bifurcation point. The insets in $(\mathrm{a}, \mathrm{c})$ show details around $\mathrm{FP}^{D}$ and $\mathrm{FP}^{D}{ }^{D}$. The coloured vertical broken lines correspond to the parameter values used to obtain the simulation results in Figure 3. Parameter values as in Tables A.1 and A.2 except for $\gamma=0.4 \mu \mathrm{M}^{-1} \mathrm{hr}^{-1}$. 
exist for the Cyclin D dependent ATP release model variant (Figure 3c) and only stable torus solutions exist for the $R_{\mathrm{S}}$ dependent ATP release model (Figure 3d), the maximal Cyclin D concentration of the second cell is high enough for it to be considered as having left quiescence and embarked on the cell cycle. However, it is clear that the cell cycles of the two cells are not synchronised for both model variants. For the asynchronous limit cycle solution for the Cyclin D dependent ATP release model variant (Figure 3c, blue curve) cells are phase locked almost in anti-phase where high Cyclin D concentration in cell 1 correspond to low Cyclin D concentrations in cell 2 (and vice versa). Figures $3 \mathrm{e}$ and $3 \mathrm{f}$ illustrate that when cells are identical ( $a_{\mathrm{d} 2}^{\prime}=a_{\mathrm{d} 1}^{\prime}=0.41$ ) synchronised limit cycle solutions exist for both model variants. An additional stable asynchronous solution also exists for the Cyclin D dependent ATP release model (Figure 3e, blue curve) where cells oscillate almost in anti-phase with each other. The fact that it is not always guaranteed that the system will evolve to a synchronous solution may seem surprising. However, there are a number of examples in the literature of where identical coupling between oscillators to that considered in this work leads to nonsynchronous behaviour. In the field of electronic engineering, Kitajima et al. (1998) demonstrated that a system of two oscillatory ordinary differential equation models for voltage ports permits torus solutions. Furthermore it has been demonstrated that a system of two coupled van der Pol oscillators and, separately, a system of two coupled Morris-Lecar oscillators permit the coexistence of stable synchronous and stable anti-phase phase locked solutions for certain parameter values (Postnov et al., 1999).

The results in Figures 2 and 3 illustrate that synchronous solutions only exist for a small range of the parameter space $\left(0.4086<a_{\mathrm{d} 2}^{\prime}<0.411\right.$ in Figures $2 \mathrm{a}$ and $2 \mathrm{c}$ and for $0.403<a_{\mathrm{d} 2}^{\prime}<4.17$ in Figures $2 \mathrm{~b}$ and $\left.2 \mathrm{~d}\right)$ which correspond to cells with nearly identical parameter values (i.e. where $a_{\mathrm{d} 2}^{\prime} \approx a_{\mathrm{d} 1}^{\prime}$ ). Even then, for the Cyclin D dependent mode variant, these solutions coexist with stable limit cycle solutions where two cells cycle almost in anti-phase with each other. In reality cells of the same type exhibit small differences and therefore we can not expect synchronous dynamics to dominate cell cycle timings over the long term. However, the possibility remains that in the short term, before the system attains its asymptotic state, cells cycle in near synchrony. We have shown that cycling radial glia can recruit otherwise quiescent cells onto the cell cycle during neurogenesis (Barrack et al., 2014). This opens up the possibility that quiescent cells recruited could cycle in synchrony with already cycling cells for the relatively short period of neurogenesis. This would ensure that, within a cohort of cells, all daughter cells created by proliferating radial glia would be born at the same point in time. For this scenario to be feasible, quiescent cells would need to be recruited on to the cell cycle quickly in order for this 
(a)

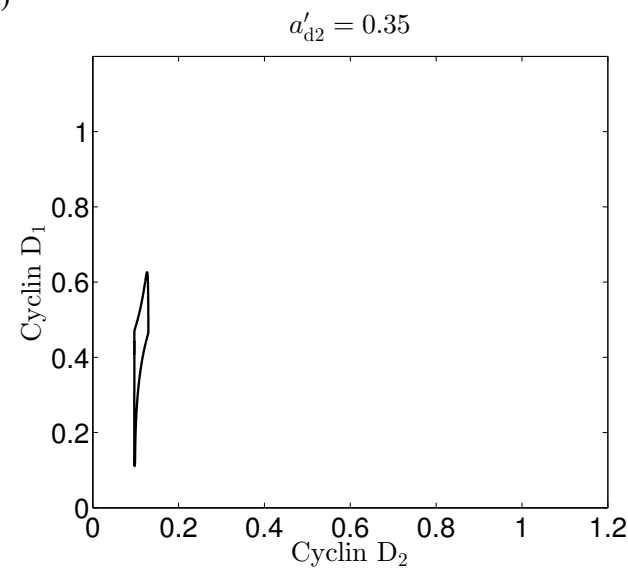

(c)

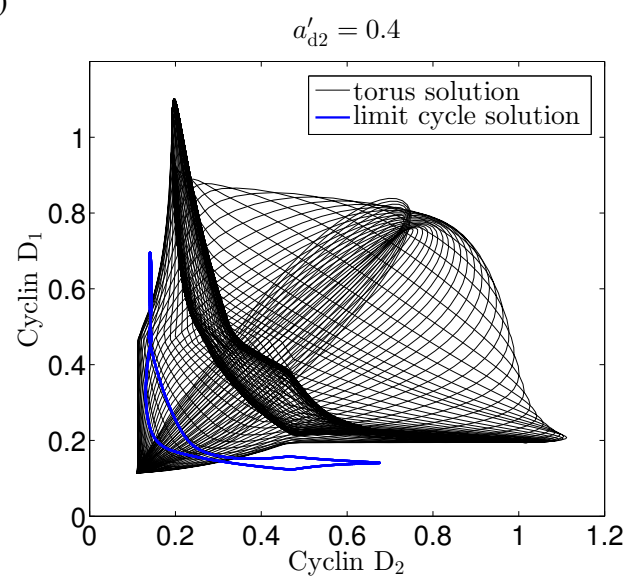

(e)

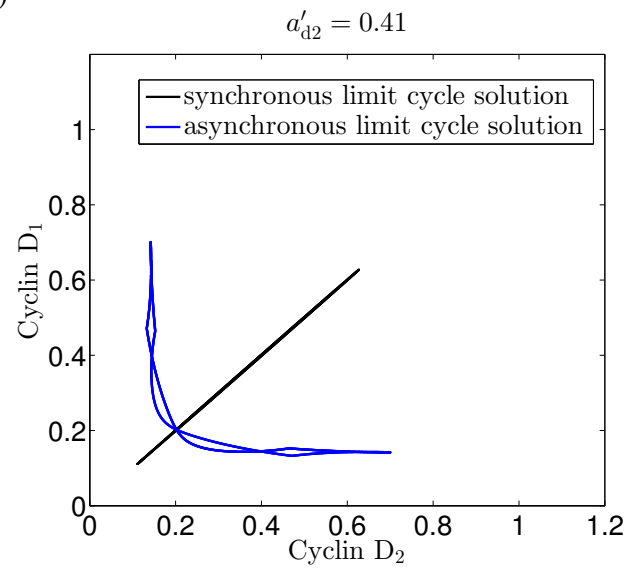

(b)
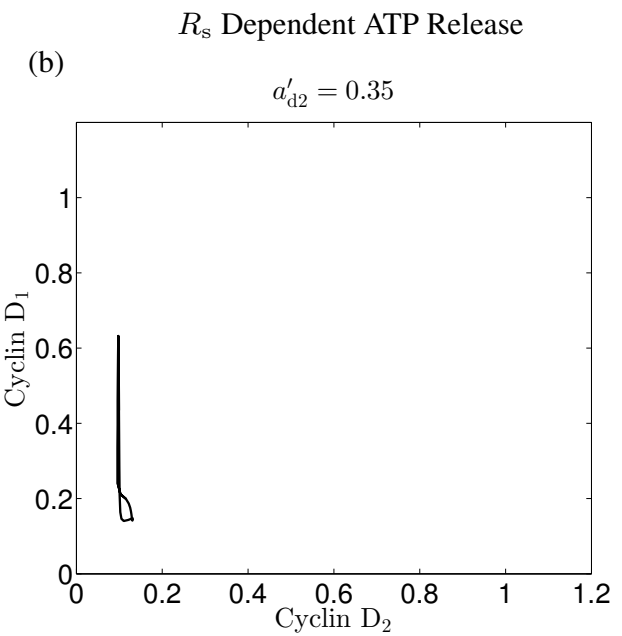

(d)

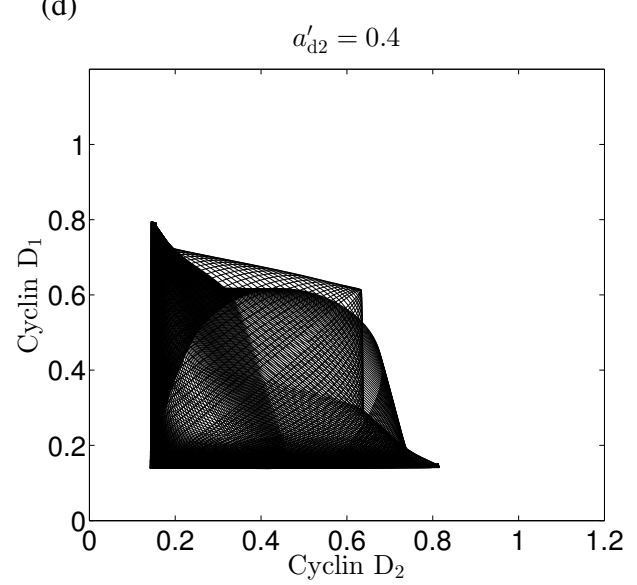

(f)

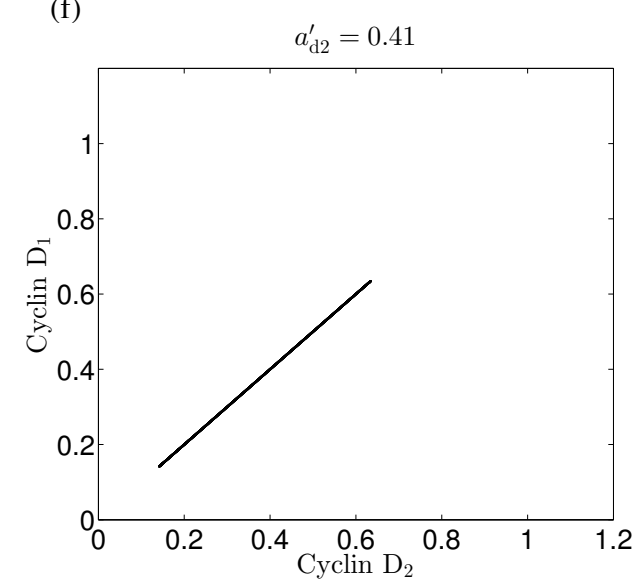

Figure 3: Simulation results showing the different oscillatory solutions uncovered in the bifurcation analysis of two coupled radial glial cells. Parameter values used correspond to the vertical coloured lines in Figure 2. (a,b) show limit cycle solutions with low maximal Cyclin D concentrations in cell 2. (c) shows torus and limit cycle solutions for the Cyclin D dependent model variant (which coexist and were obtained from different sets of initial conditions) with high maximal Cyclin D concentrations for cell 2. (d) shows a torus solution for the $R_{\mathrm{S}}$ model variant. (e) shows synchronous and asynchronous limit cycle solutions (which coexist). (f) shows a synchronous limit cycle solution for the $R_{\mathrm{S}}$ dependent ATP release model variant. All simulations were run for a total of 2000 hours of simulation time and the transients are not shown. Parameter values as in Tables A.1 and A.2 except for $\gamma=0.4 \mu \mathrm{M}^{-1} \mathrm{hr}^{-1}$. 
process to account for the large increase in proliferation associated with ATP. Motivated by this we investigate the time it takes for a quiescent cell to be recruited on to the cell cycle as a function of the calcium coupling strength parameter and the zero calcium Cyclin D synthesis rate $a_{\mathrm{d} 2}^{\prime}$ of the quiescent cell. Figures $4 \mathrm{a}$ and $4 \mathrm{~b}$ show cell cycle recruitment of a quiescent cell by a cycling cell. A quiescent cell is regarded to have been recruited when its Cyclin D concentration reaches 0.4 and where all subsequent oscillations have a maximal Cyclin D concentration above 0.4. A Cyclin D value of 0.4 is consistent with with the limit cycle solutions in (Obeyesekere et al., 1999) that correspond to the cycling state. The initial conditions of the quiescent cell are such that, in the absence of intercellular coupling, the cell will remain at a fixed point solution corresponding to dormancy and will not oscillate independently of an externally driven perturbation. In contrast the initial conditions of the driving cell are such that it will oscillate irrespective of any extracellular coupling to the quiescent cell. This gives a physically realistic scenario whereby a quiescent cell will remain dormant unless recruited by an already cycling cell. Figures $4 \mathrm{c}$ and $4 \mathrm{~d}$ indicate that cell cycle recruitment is ensured for the higher values of the Cyclin D synthesis rate $a_{\mathrm{d} 2}^{\prime}$, irrespective of the value for the calcium coupling strength $\gamma$. The average time to recruitment in Figure 4c is 1.007 hours and it is 0.7446 hours in Figure 4d. These times are consistent with the notion that the cell cycle recruitment time is extremely short and makes up a small fraction of the total period of neurogenesis in mammals which lasts 6 days in rat embryos and an estimated 10 weeks in human embryos (Bayer et al., 1993; Caviness et al., 1995; Coti Bertrand et al., 2006).

For $\gamma>4.5$ in Figure $4 \mathrm{c}$ and for $\gamma>4$ in Figure $4 \mathrm{~d}$ the relationship between the time to recruitment, the Cyclin D synthesis rate of the quiescent cell $a_{\mathrm{d} 2}^{\prime}$ and calcium coupling strength $\gamma$ is monotonic. Below these values there are regions of parameter space where the relationship is not monotonic (some of these are circled in red in the plots). This is due to the different transient dynamics of the system under the parameter regimes within these regions. To illustrate this, we plot the Cyclin D concentrations of both cells for the $R_{\mathrm{S}}$ dependent ATP release model where $a_{\mathrm{d} 2}^{\prime}=0.08$ and where $\gamma=2.15,2.5$ and 2.8 in Figure 5. The entrainment time (marked with a red dot) is 137.4 hours when $\gamma=2.15$ (Figure 5a). It falls to 110.1 hours when $\gamma=2.5$ (Figure $5 \mathrm{~b}$ ) and then rises to 134.95 hours when $\gamma=2.8$ (Figure 5c). Note, in Figures $5 \mathrm{~b}$ and c, the Cyclin $\mathrm{D}$ concentration of the quiescent cell reaches 0.4 (denoted by a green dot in each plot) prior to the point of entrainment. However because the maximal Cyclin D concentrations of subsequent oscillations are below 0.4 (labelled in both plots), these points do not satisfy the definition of entrainment. 
Cyclin D Dependent ATP Release

a)

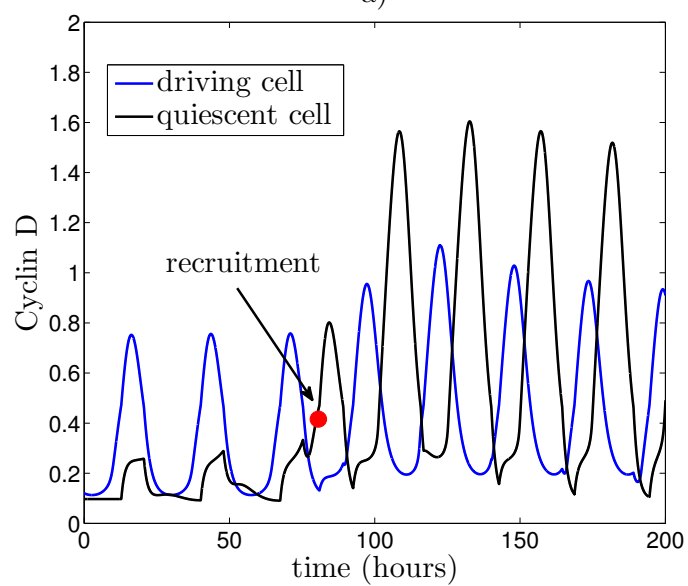

c)

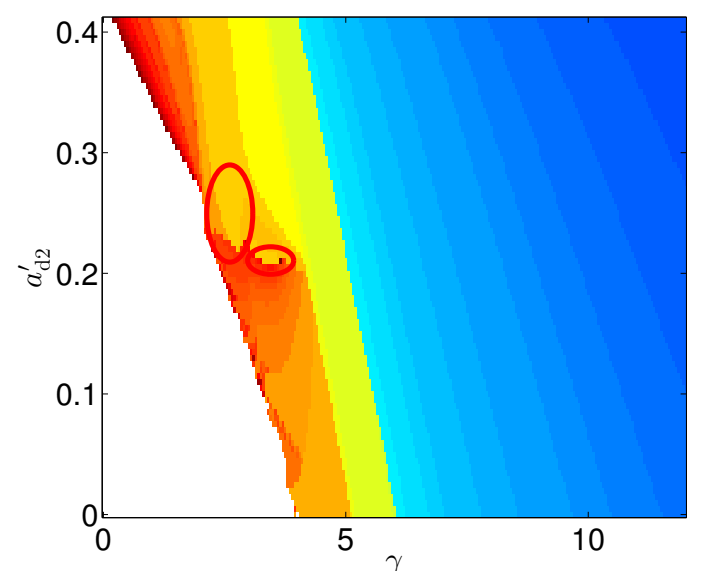

$R_{\mathrm{S}}$ Dependent ATP Release

b)

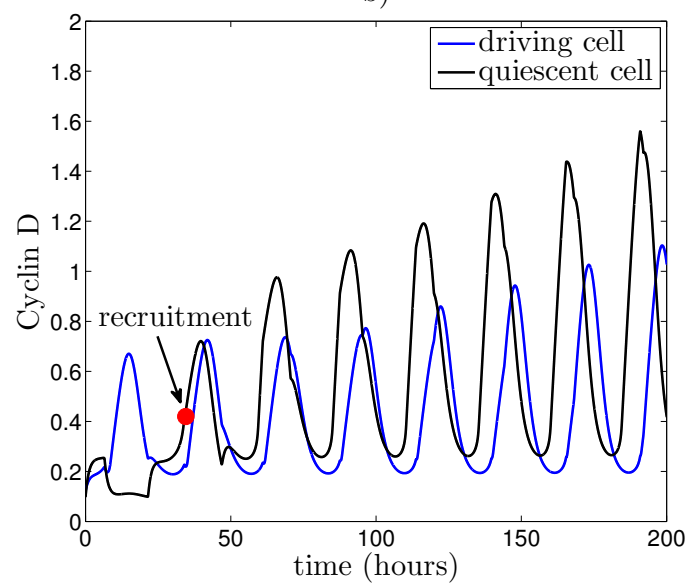

d)

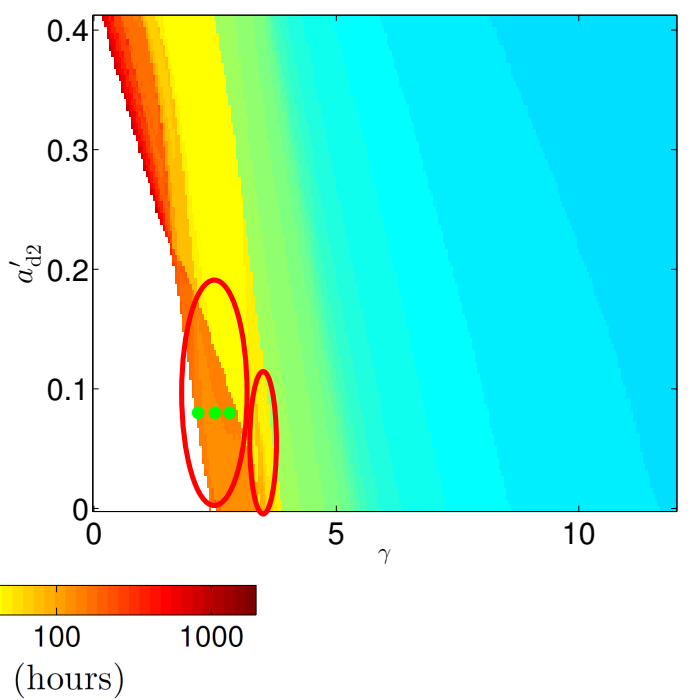

time to recruitment (hours)

no recruitment

Figure 4: $(a, b)$ show examples of cell cycle recruitment of a quiescent cell by a driving cell for both model variants. The point of entrainment where the Cyclin D value of the quiescent cell reaches 0.4 (and where the maximal Cyclin D concentrations of all subsequent oscillations exceeds 0.4) is marked by a red dot. (c,d) show the times of entrainment as a function of the calcium coupling strength $\gamma$ in both cells and the intrinsic Cyclin D synthesis rate of the quiescent cell $a_{\mathrm{d} 2}^{\prime}$. White corresponds to instances when the quiescent cell was not recruited by the end of the simulation which lasted 2000 hours. Some of the areas where the relationship between entrainment time and the control parameters are not monotonic are circled in red and are discussed in the text. The three green dots in (d) correspond to the parameter values used to obtain the simulation results in Figure 5. Parameter values as in Tables A.1 and A.2 except $a_{\mathrm{d} 2}^{\prime}=0.35$ in $(\mathrm{a}, \mathrm{b})$. 

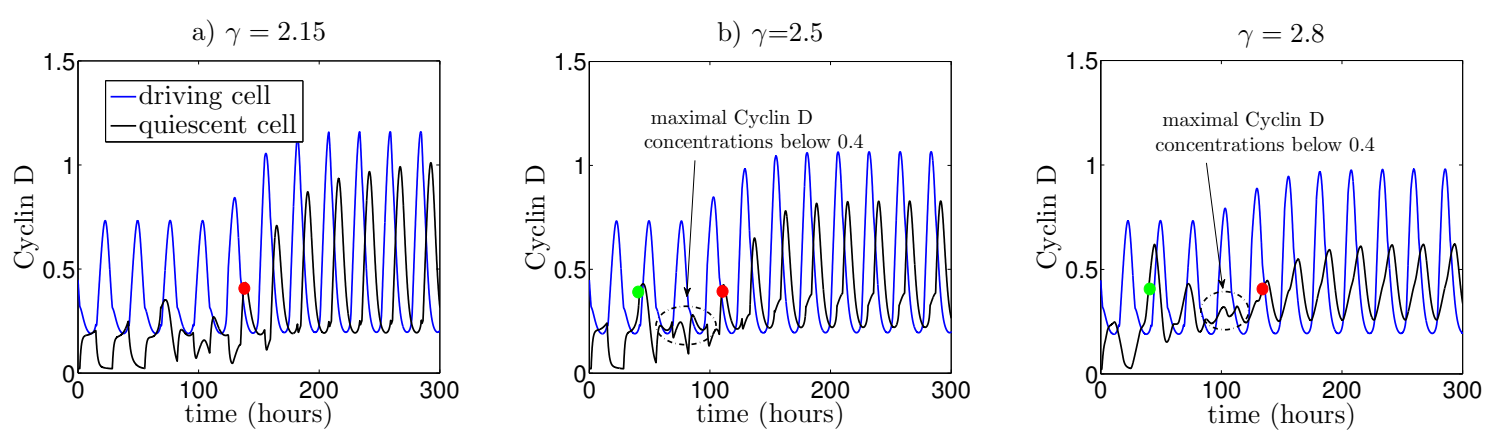

Figure 5: Simulation results for the $R_{\mathrm{S}}$ dependent ATP release model which illustrate that for some regions of the parameter space of Figure 4 the relationship between the entrainment time and calcium coupling strength $\gamma$ is not monotonic. The point at which the initially quiescent cell is entrained on to the cell cycle is marked with a red dot in each plot. In (b) and (c) the green dot denotes the first time at which the Cyclin D concentration of the quiescent cell reaches 0.4. However, this point does not correspond to entrainment as the maximal concentrations of subsequent oscillations are below 0.4. Parameter values as in Tables A.1 and A.2 except $a_{\mathrm{d} 2}^{\prime}=0.08$.

Next, we turn our attention to measuring cell cycle synchrony in cohorts made up of initially dormant and initially cycling cells during the transient period of neurogenesis. To determine the degree of cell cycle synchronisation, we use the normalised cross-correlation coefficient. This has been used extensively to measure synchrony between oscillators, particularly in neuroscience (Wang, 1995; Buzsáki and Draguhn, 2004) and for two cells $j$ and $k$ is defined as

$$
C\left(x_{j}(t), x_{k}(t)\right)=\frac{\int_{T_{1}}^{T_{2}}\left(x_{j}(t)-\bar{x}_{j}(t)\right)\left(x_{k}(t)-\bar{x}_{k}(t)\right) d t}{\sqrt{\int_{T_{1}}^{T_{2}}\left(x_{j}(t)-\bar{x}_{j}(t)\right)^{2} d t} \sqrt{\int_{T_{1}}^{T_{2}}\left(x_{k}(t)-\bar{x}_{k}(t)\right)^{2} d t}},
$$

where $x_{j}(t)$ is one of the model variables (e.g. Cyclin $\mathrm{D}\left(D_{j}\right)$, Cyclin $\mathrm{E}\left(E_{j}\right)$ etc.) of cell $j$ and $\bar{x}_{j}(t)$ is the mean of $x_{j}(t)$ which is given by $\frac{1}{T_{2}-T_{1}} \int_{T_{2}}^{T_{2}} x_{j}(t) . C\left(x_{j}(t), x_{k}(t)\right) \in[-1,1]$ will take a value of $1(-1)$ if the two cells cycle synchronously (in anti-phase) between $T_{1}$ and $T_{2}$. The higher the value of $C\left(x_{j}(t), x_{k}(t)\right)$ the greater the level of synchrony between cells $j$ and $k$. To determine the overall synchrony in a cohort of more than two cells we use the following expression

$$
C(\mathbf{x})=\frac{1}{N(N-1)} \sum_{\substack{j=1 \\ j \neq k}}^{N} \sum_{\substack{k=1 \\ j}}^{N}\left(x_{j}(t), x_{k}(t)\right) \quad x_{j}(t), x_{k}(t) \in \mathbf{x}
$$

where $\mathbf{x}=\left\{x_{1}(t), x_{2}(t), \ldots, x_{N}(t)\right\}$ and $N$ is the number of cells in the cohort. $T_{1}$ in equation (1) corresponds to the time at which the Cyclin D value of the final quiescent cell to be recruited reaches 0.4 indicating all cells 
within the cohort are cycling. We choose a value of 840 hours for $T_{2}$ which is consistent with the period of neurogenesis in mammals (Bayer et al., 1993; Caviness et al., 1995; Coti Bertrand et al., 2006).

To determine if our model permits near synchronous entrainment of quiescent cells, and which of the two variants has the greater propensity for synchronous entrainment, we calculate the cohort correlation coefficient (equation (2)) under a number of different cases for multicellular systems coupled on one dimensional and two dimensional lattices with zero flux boundary conditions. To ensure every case is physically realistic, as for the results in Figure 4, the initial conditions of all quiescent cells are such that, in the absence of intercellular coupling, they exhibit stable fixed point solutions which corresponds to quiescence. The cases are described below.

Case 1. Ten cell cohort (one spatial dimension), one cell initially cycling, nine cells initially dormant. Spatial position of the cycling cell varied. Here we investigate the impact that the position of an initially cycling cell within the one dimensional cohort has on overall synchrony. Three subcases are considered. In subcase 1a), we choose cell 1 (counting sequentially from the left to right along the lattice, see Figure 6). For subcases 1b) and 1c) cells 3 and 5 are chosen as the cycling cells respectively.

Case 2. Ten cell cohort (one spatial dimension), where the number of initially cycling cells and quiescent cells is varied. To investigate the affect of varying the number of initially cycling cells on synchrony we consider four subcases, 2a) where only cell 1 is initially cycling (note, this subcase is identical to subcase 1a) described above), $2 \mathrm{~b}$ ) where both cells 1 and 2 cycle, $2 \mathrm{c}$ ) where cells 1-3 cycle and finally $2 \mathrm{~d}$ ) where cells 1-4 cycle. All cycling cells have the same initial conditions.

Case 3. Ten cell cohort (one spatial dimension), where initially cycling cells are distributed between quiescent cells. Here we investigate the impact that distributing initially driving cells amongst dormant cells has on synchrony. Three subcases are considered, 3a) where cells 1,2,3 are cycling (note, this is identical to subcase 2c)), 3b) where cells 1,3 and 5 are cycling and 3c) where cells 2,5 and 8 cycle. All cycling cells have the same initial conditions.

Case 4. Ten cell cohort (one spatial dimension), where the initial conditions of the cycling cells are varied. 


\section{a) Cell 1 initially cycling}

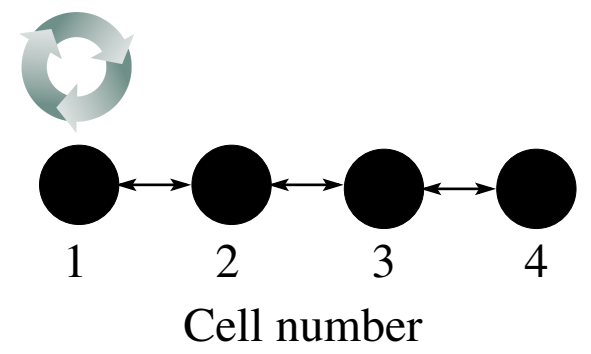

b) Cell 2 initially cycling

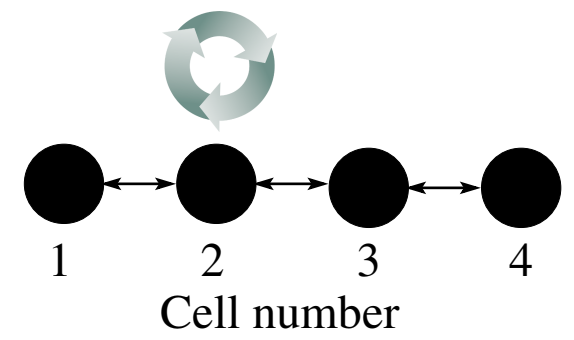

Figure 6: Schematic showing cohorts of four cells each coupled to their nearest neighbours with zero flux boundary conditions. Cells are numbered sequentially from left to right with a) cell 1 initially cycling and b) cell 2 initially cycling.

Here, cycling cells are initialised at different phases of the cell cycle, in order to gain an understanding of how this affects synchronisation. Cells 1,2 and 3 are chosen as the initially cycling cells. Cell 3 is initialised at a more advanced point in the cell cycle than cell 2 and cell 2 is more advanced than cell 1 . Phase differences of 0 (identical to subcase 2c)), 1, 3 and 5 hours between adjacent cycling cells are considered.

Case 5. Cohorts with more than ten cells (one spatial dimension). Here, systems of ten, twenty and thirty cells are simulated so that it can be determined what impact larger systems of cells may have on synchrony. Cells 1-4 are chosen as the cycling cells and all have the same initial conditions.

Case 6. Cohorts with 25 cells (two spatial dimensions). To investigate the dependency of synchrony on the spatial dimension we consider a $5 \times 5$ two dimensional cohort of cells. The cell in the centre of the cohort is chosen as the initially cycling cell with all other cells initially quiescent.

To account for slight physiological differences between cells, for each subcase, a number of parameter regimes are considered where no two cells have the same parameter values. For each simulation we first generate $N$ (where $N$ is the total number of cells in the cohort) vectors $\mathbf{P}_{\mathbf{j}}=\left(P_{1, j}, \ldots, \lambda_{j} P_{l, j}, \ldots, P_{42, j}\right)$ where the $P_{l, j}, l=1, \ldots, 42$, refer to the baseline parameter values, as indexed in Tables A.1 and A.2, corresponding to cell $j=1, \ldots, N$. For each value of $l$, we set $\lambda_{j}=1+\Phi_{j}$ where $\Phi_{j}$ is uniformly distributed on [-0.4, 0.4]. Five simulations are run for each value of $l$. As there are 41 different parameter values for each model variant this gives a total of 205 simulations for each subcase. Note, the global parameters for ATP diffusion $D_{\text {ATP }}$ and the distance between cells $\Delta x$ indexed by 43 and 44 respectively are fixed in every simulation as 
(a)

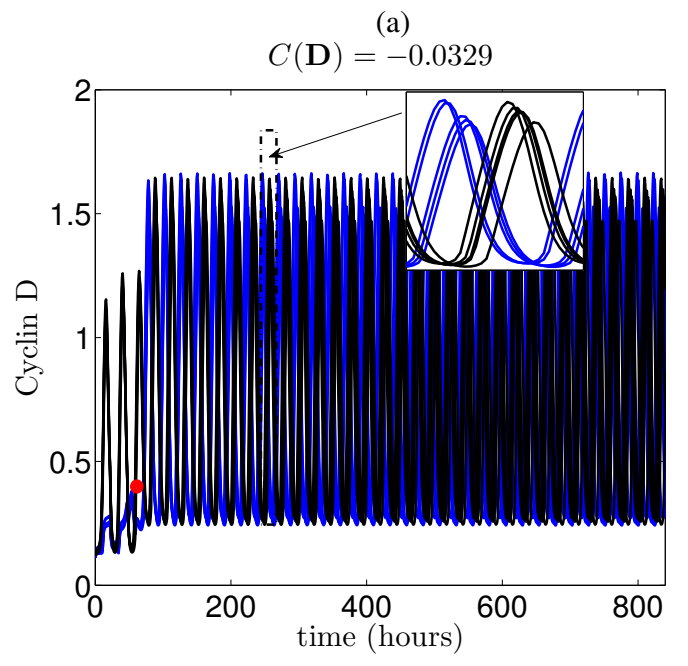

Ten cell systems

(b)

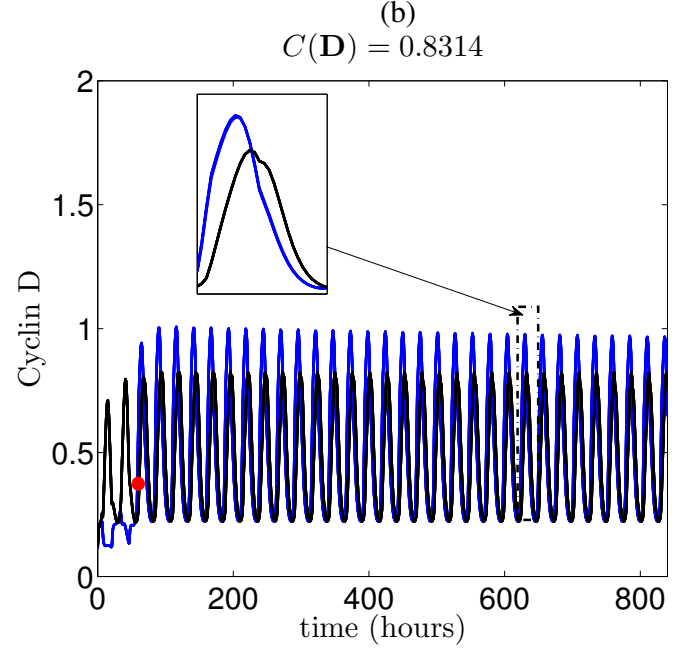

Figure 7: Simulations of ten cell systems showing entrainment of quiescent cells by driving cells (point of entrainment of final quiescent cell recruited on the cell cycle is marked with a red dot). (a) Entrainment is asynchronous for the Cyclin D dependent model variant and (b) almost synchronous for the $R_{\mathrm{S}}$ model variant. The insets show details around some of the oscillations. The intrinsic Cyclin D synthesis rate $a_{\mathrm{d} j}^{\prime}$ of cell $j$, was chosen as the parameter to perturb by a random amount (see text for details). Cells 1-5 were chosen as the cycling cells and all have identical initial conditions. Cohort cross-correlation scores $C(\mathbf{D})$ are given in the figure titles.

these parameters cannot be regarded as proxies for physiological differences in the cells. Before presenting the results of the simulations an example of cell cycle recruitment in a system of ten cells is shown in Figure 7. The time to recruitment of the final quiescent cell to be entrained on the cell cycle (which corresponds to $T_{1}$ from equation (1)) is marked in red. For the Cyclin D dependent ATP release model variant (Figure 7a) the driving cells (blue curves) are cycling almost in synchrony with each other and the initially quiescent cells (black) are cycling almost in synchrony with one another. However, relative to each other, the quiescent and driving cells are cycling almost in anti-phase. This observation is reflected by the cohort cross correlation score which is close to 0 . Driving cells cycle almost in synchrony with quiescent cells for the $R_{\mathrm{S}}$ dependent ATP release model variant (Figure $7 \mathrm{~b}$ ) and consequently the cohort cross correlation score is close to 1 .

Distributions of the cohort cross-correlation coefficients obtained from the 205 simulations for each subcase are given in Figure 8. For case 1 where one cell is initially cycling and all others are quiescent (Figures 8a-c) cross-correlation coefficient scores are close to 1 for the $R_{\mathrm{S}}$ dependent model variant, where ATP is released at $\mathrm{G}_{1} / \mathrm{S}$ phase transition of the cell cycle. In contrast scores for the Cyclin D dependent model variant are smaller. 
Therefore, for this case, release at the $\mathrm{G}_{1} / \mathrm{S}$ phase transition has a greater propensity to give rise to synchronous entrainment of quiescent cells than the Cyclin D dependent model where release occurs during mid $\mathrm{G}_{1}$ phase. The reason for this is the delay before the quiescent cells embark on the cell cycle after they have received an ATP mediated calcium signal from the driving cell. For the $R_{\mathrm{S}}$ dependent model variant ATP is released after a Cyclin D peak and causes an increase in calcium and ultimately Cyclin D in the quiescent cell. However, there is a delay before the Cyclin D concentration is high enough to push the quiescent cell on to the cell cycle. This delay coincides with the time it takes for the driving cell's Cyclin D concentration to rise again after its minimum. Hence both the driving and quiescent cells will initially be cycling in, or near, synchrony with each other. For the Cyclin D dependent ATP model variant, ATP release begins when the driving cell approaches its Cyclin D peak. After receiving this ATP signal, the quiescent cell will begin to cycle. However, because of the delay before it embarks on the cell cycle, this point will coincide with when the Cyclin D concentration of the driving cell is falling and approaching a minimum. Consequently the cells will be initially cycle out of phase with each other. It is also noticeable that the results in Figures 8a-c are almost identical and therefore the spatial location of the driving cell has no meaningful impact on cell cycle synchronisation. This is the case because the timescale for spatial diffusion of ATP across cohorts made up of a small number of cells is of the order of seconds and is extremely fast compared to the time scale of the cell cycle which is of the order of hours. Given a diffusion time of $t=\frac{\Delta x^{2}}{2 D_{\mathrm{ATP}}}$ (Einstein, 1905), it will take approximately 16.7 seconds for ATP to diffuse from one end of a ten cell cohort to the other. Therefore, within a cohort made up of a small number of cells, no mater where the driving cell lies the ATP it releases will diffuse through the entire extracellular space of the cohort in an extremely short space of time. This means that all quiescent cells within the cohort will get recruited on to the cell cycle almost at the same time.

For case 2 where the impact of varying the number of initially cycling cells is investigated it is again clear from Figures 8d-g that the cohort synchrony coefficients for $R_{\mathrm{S}}$ dependent model variant are close to 1 , indicating near synchrony, and are larger than the corresponding coefficients for the Cyclin D dependent ATP release model variant. Furthermore, as the number of driving cells increases (and correspondingly the number of quiescent cells falls) the cross-correlation falls, although the fall for the $R_{\mathrm{s}}$ dependent model is more modest than for the Cyclin D dependent model. The reason for the fall is related to the ratio of driving cells to quiescent cells within the cohort. Because extracellular diffusion of ATP is so fast accross the cohort, every quiescent cell receives an ATP signal almost at the instant at which it is released from the driving cell or cells. Consequently, 
all cells are recruited almost at the same time and will, initially at least, cycle in synchrony with each other. Therefore the greater the proportion of quiescent cells within the cohort the greater the proportion of cells that will be cycling in synchrony. Hence for Figure 8d, where the ratio of quiescent cells to driving cells is 9 to 1 , the cohort synchrony score is higher than it is in Figure $8 \mathrm{~g}$ where the ratio is 6 to 4 .

For the third case (Figures 8h-j) it is clear that the spatial distribution of three initially cycling cells has no discernible impact on the cohort synchrony score. This is because ATP diffuses through extracellular space so quickly. For all subcases considered, the synchrony scores for the $R_{\mathrm{S}}$ dependent model variant are higher than for the Cyclin D dependent ATP release variant.

For case 4 where the initial phase difference between the cells is varied (Figures $8 \mathrm{k}-\mathrm{n}$ ), again the $R_{\mathrm{S}}$ dependent model gives higher values for the synchrony score in all subcases than the Cyclin D dependent model variant. A change in the phase difference has little to no effect on the results because it is the behaviour of the quiescent cells which outnumber the driving cells 7 to 3 which contribute most to the synchrony score. In such a small system these will be recruited at the same time irrespective of the phase difference between the driving cells and will initially begin to cycle in synchrony with each other. Additionally, for the $R_{\mathrm{S}}$ dependent model quiescent cells will cycle in near synchrony with the driving cells whilst, for the Cyclin D dependent model, they will cycle in anti-phase which is why the synchrony scores for the $R_{\mathrm{s}}$ dependent model are higher.

For the case 5 where the number of cells in the system is varied (Figures 8o-q) the synchrony scores for the $R_{\mathrm{S}}$ dependent model are higher than those for the Cyclin D dependent model. Furthermore, the synchrony scores increase with the number of cells in the system. This is because even though the number of cells is increasing the number of driving cells is fixed and hence the ratio of quiescent cells to driving cells is increasing with the total number of cells in the system. As noted above the proportion of quiescent cells in the system have a large impact on synchrony scores with greater proportions leading to higher scores. It is this phenomenon that is seen here.

For the final case where a two dimensional cohort is considered (Figure 8r), the results are similar in the sense that the synchrony scores for the $R_{\mathrm{S}}$ dependent model are close to 1 and are higher than those for the Cyclin D dependent model. The scores for each model variant are also higher than the respective scores of all 
other subcases. This is due to the greater number of nearest neighbour cells in two dimensions compared to one dimension (4 compared to 2 in our model, equation (A.11)). Consequently, where a driving cell would recruit two neighbours in synchrony in a one dimension it will recruit four in two spatial dimensions. It is this which leads to higher synchrony scores.

A further observation is that the oscillatory solutions uncovered in Figure 2 for two cells persist in multicell systems. In particular, solutions consistent with torus and limit cycle solutions (both synchronous and asynchronous) were encountered. For asynchronous limit cycle and torus solutions, despite the fact that cells eventually fall out of synchrony, this occurs extremely slowly relative to the time scale of neurogenesis. Furthermore, if recruited cells initially cycle in synchrony with the driving cells (as is the case for the $\mathrm{R}_{s}$ dependent model variant), then they will remain synchronised for the duration of the period of neurogenesis. To illustrate this, we calculated the cohort synchrony score (equation (2)) in sliding windows of 60 seconds and plotted this as a function of time for 10 cell systems which exhibited behaviour consistent with asynchronous solutions. These results are shown in Figure 9. For the Cyclin D model variant (Figure 9a) the synchrony score is close to 0.53 and remains at this value for just over 2000 hours before falling rapidly for the remaining simulation time. For the $\mathrm{R}_{s}$ dependent model variant (Figure $9 \mathrm{~b}$ ) the synchrony score is initially above 0.85 and remains so for at least 2000 hours before falling sharply. In the plots the 840 hour mark, which indicates the end of neurogenesis, is marked with a broken red line. Clearly the synchrony score for the $\mathrm{R}_{s}$ dependent model is above 0.85 during the entirety of this period indicating that recruited cells cycle in near synchrony with the driving cell during neurogenesis even when synchronous solutions do not exist.

\section{Discussion}

In this paper, we addressed the question of whether an ATP mediated calcium mechanism can sustain synchronous cycling of proliferating radial glia. We took an existing model for the coupling between ATP mediated calcium release and the cell cycle of a single radial glia and considered populations of radial glia coupled via the diffusion of extracellular ATP. Bifurcation analysis of two cell systems indicate that stable synchronous oscillatory solutions are far from guaranteed with a number of asynchronous solutions also existing. Despite this, simulation results suggest that it is not the asymptotic behaviour of the system which is important but the transient behaviour in order to account for cell cycle synchronisation during neurogenesis in our model. In 
Case 1 - Spatial position of initially cycling cell varied

a) Cell 1 initially cycling

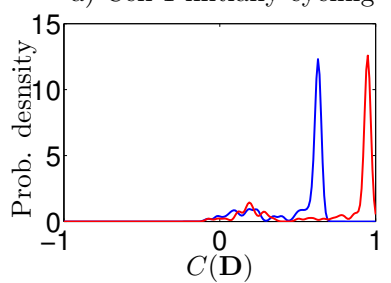

b) Cell 3 initially cycling

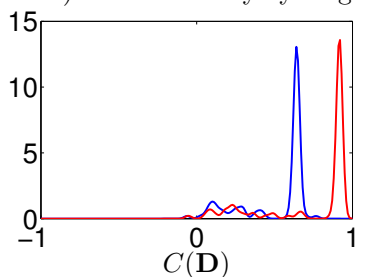

c) Cell 5 initially cycling

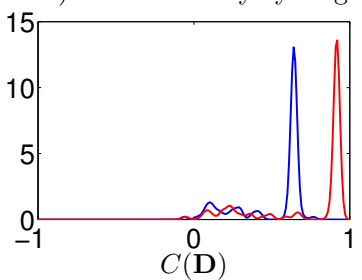

d) Cell 1 initially cycling

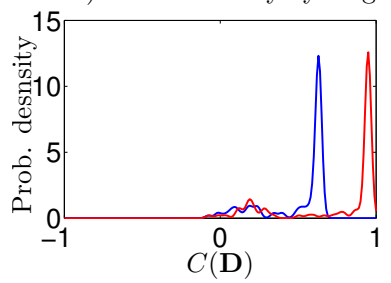

Case 2 - Number of initially cycling cells varied

e) Cells 1 and 2 cycling

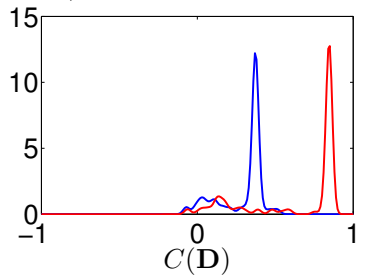

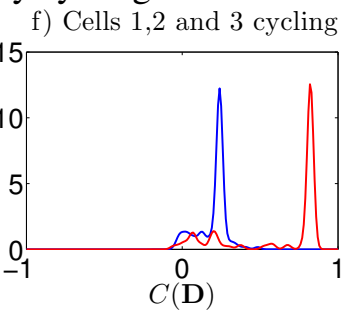

g) Cells 1,2,3 and 4 cycling

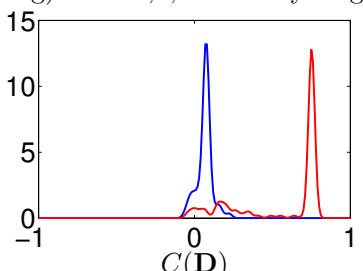

Case 3 - Spatial distribution of initially cycling cells varied h) Cells 1,2 and 3 cycling
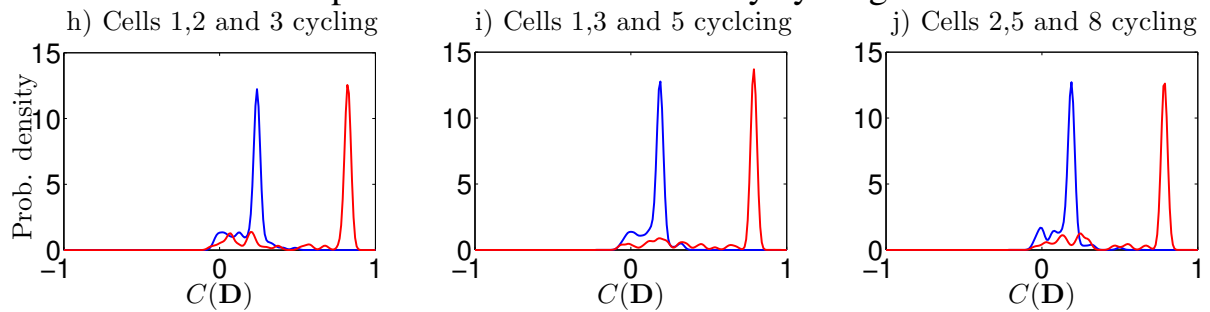

Case 4 - Initial phase difference of cycling cells varied

k) Phase difference - 0

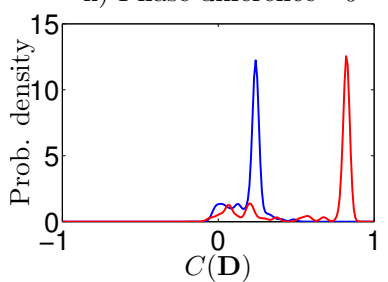

Case 5 - Total number of cells varied

o) Ten cells

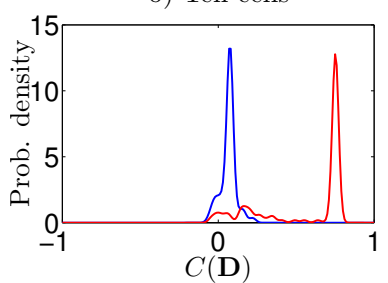

l) Phase difference - 1 hour
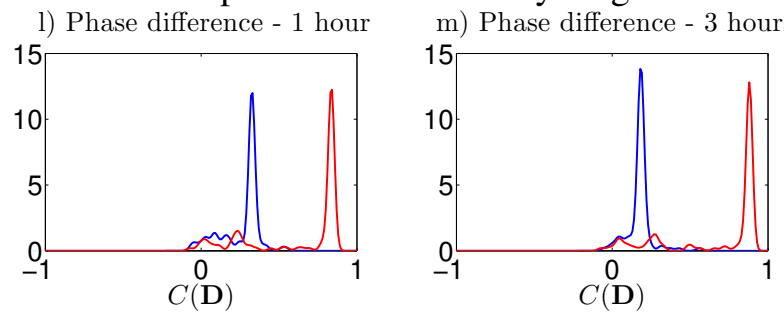

q) 30 cells
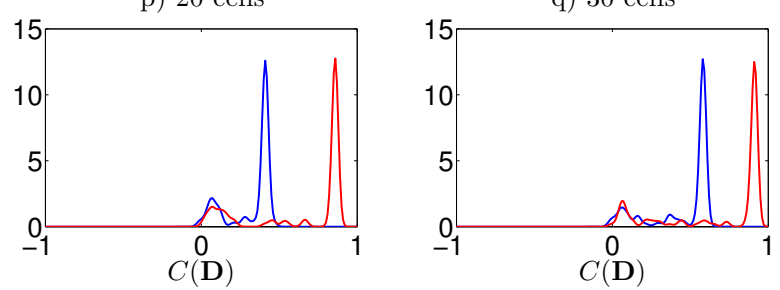

n) Phase difference - 5 hours

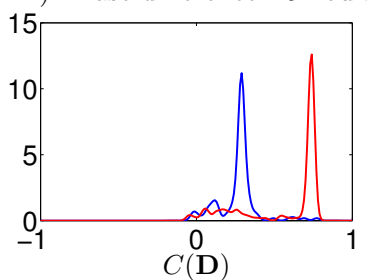

Case 6 - 2D

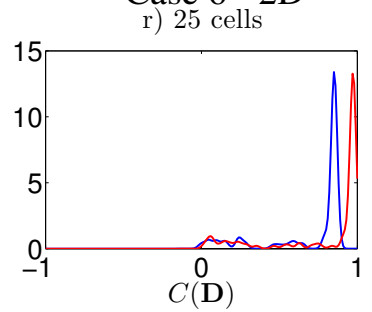

Legend

Cyclin D dependent ATP release

$R_{s}$ dependent ATP release

Figure 8: Distributions of the cohort cross correlation scores (equation (2)) obtained for every case which indicate that the $R_{\mathrm{S}}$ dependent ATP release model has a greater propensity to give rise to synchronous or near synchronous solutions when compared to the Cyclin D dependent ATP release model. Distributions obtained by taking kernel density estimates (Silverman, 1986) with a bandwidth of 0.02 . Results where entrainment failed, or where under the parameter regime initially quiescent cells cycle independently of an ATP signal from a driving cells, are not included. 
Cyclin D Dependent ATP Release

(a)

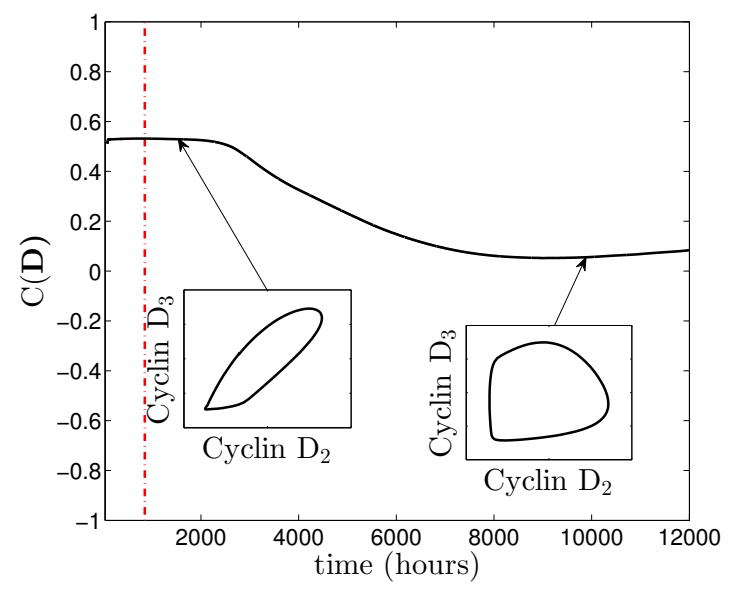

$R_{\mathrm{S}}$ Dependent ATP Release

(b)

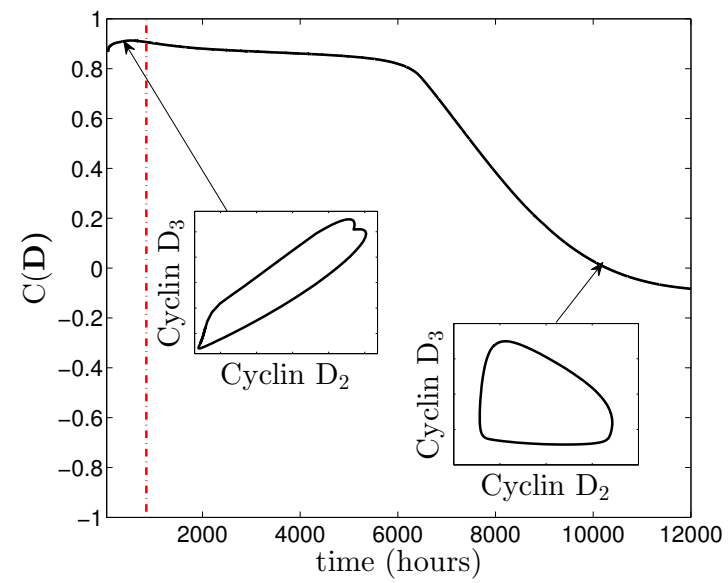

Figure 9: Plots illustrating that, even when stable synchronous solutions do not exist, recruited quiescent cells will cycle in synchrony for the duration of neurogenesis. The synchrony score (equation (2)) for a cohort of ten cells is plotted as a function of time for both model variants. The broken red line corresponds to the end of the period of neurogenesis. The insets show Cyclin D for cells 2 and 3 near the beginning of the simulation where cells are closer to synchrony and towards the end of the simulation time where cells are further away from synchrony. Cell 1 was chosen as the driving cell with all other cells initially quiescent. Parameter values as in Tables A.1 and A.2 except for $K_{\mathrm{R} 1-10}=$ $\{17.29 \mu \mathrm{M}, 33.98 \mu \mathrm{M}, 23.99 \mu \mathrm{M}, 26.56 \mu \mathrm{M}, 23.16 \mu \mathrm{M}, 19.74 \mu \mathrm{M}, 33.06 \mu \mathrm{M}, 26.47 \mu \mathrm{M}, 15.05 \mu \mathrm{M}, 27.34 \mu \mathrm{M}\}$. 
particular, ATP release by already cycling cells during the $G_{1} / S$ phase transition of the cell cycle is sufficient to recruit quiescent cells onto the cell cycle which then cycle in near synchrony with the driving cells for the duration of neurogenesis. In contrast although ATP release during mid $\mathrm{G}_{1}$ phase leads to the recruitment of quiescent cells, the cells have a far greater propensity to cycle asynchronously. Furthermore, our results indicate that, within a cohort of a small number of cells, as ATP signals diffuse through extracellular space so quickly only a handful of cycling cells are required to recruit a relatively large number of quiescent cells. Indeed, because all quiescent cells are recruited almost at the same time (at which point they begin cycling in synchrony with each other) a cohort containing a large proportion of quiescent cells will have a higher network synchrony score than an equivalent system but with a lower proportion of quiescent cells. Confirming the existence of a large population of initially quiescent cells experimentally is problematic due to the difficultly in characterising $\mathrm{G}_{0}$ phase (Oki et al., 2014). However, our previously published theoretical results support the notation, first introduced in Weissman et al. (2004), that in order to account for the significant impact of ATP on proliferation rates, then initially quiescent cells are recruited on to the cell cycle by ATP signals released by already cycling cells (Barrack et al., 2014). In terms of how a large population of initially quiescent cells may arise, it has been shown, in many cell types, that the depletion of intracellular calcium leads to an accumulation of cells in the quiescent $\mathrm{G}_{0}$ state (Kahl and Means, 2003; Short et al., 1993). Furthermore it has been demonstrated in radial glia that, even with the inhibition of extracellular ATP receptors, individual cells will spontaneously release calcium from internal stores (Weissman et al., 2004). Because of the initial absence of ATP mediated calcium release we speculate that most cells will lie in $\mathrm{G}_{0}$. However, some cells will spontaneously release calcium and, due to the subsequent increase in Cyclin D levels this will cause, will begin to cycle. These cells then become the 'driving cells' which lift quiescent cells out out $\mathrm{G}_{0}$ via the ATP mediated calcium release mechanism.

In this work, we have focussed on radial glia synchronisation in cohorts of a relatively small number of cells. It is unlikely that synchrony will be global across the entire ventricular zone. As radial glia undergo interkinetic nuclear migration (Noctor et al., 2001) the cell cycle stage is linked to location within the ventricular zone. In order for the radial glial cells to physically fit in the ventricular zone it is necessary for cells to be at different stages of the cell cycle. It is therefore more likely that interspersed cohorts of radial glia will be synchronised. In principle, our model can account for this. Prior to recruitment, all quiescent cells will receive some ATP (which diffuses quickly through extracellular space) from a cycling cell. However, because ATP is degraded (equation A.10 in our model) the concentration of ATP received will depend on the proximity of the 
quiescent cell to the driving cell. Quiescent cells closer to the ATP source will receive far higher concentrations of ATP than cells further away. For the baseline parameter values of the model in a 50 cell system with all cells quiescent except cell 1 which is cycling, prior to recruitment, the concentration of ATP received by cell 2 is nearly 60 times greater than the concentration received by cell 50 (results not shown). Each driving cell has a limited range over which it can influence quiescent cells. It is conceivable that in large systems of cells, in which a number of interspersed cells are initially cycling, quiescent cells will be recruited by the driving cell to which they are closest, as they will receive a higher concentration ATP signal from this cell. This would give rise to synchronised cohorts of cells with a cohort made up of the driving cell and the quiescent cells it is able to recruit. Furthermore, having different driving cells embarking on the cell cycle at different times will lead to inter-cohort asynchrony. In Figure 10 we show simulation results for a 50 cell system for the $R_{\mathrm{S}}$ dependent model variant which shows this is possible with our model. Here, cells 12 and 38 are initially cycling at a phase difference to each other of 12 hours. All other cells are initially quiescent. The ten cells closest to the driving cells are shown and clearly, once recruited, these cycle in synchrony with their closest driving cell, giving rise to two distinct cohorts. The synchrony scores of the cohorts shown are 0.8128 and 0.8631 reflecting the fact that the cells within each cohort are synchronised. However the score after combining the cells in both cohorts is 0.0479 reflecting the fact that there is no global synchrony. Future work will investigate what conditions give rise to systems without global synchrony but where interspersed cohorts contain synchronised cells.

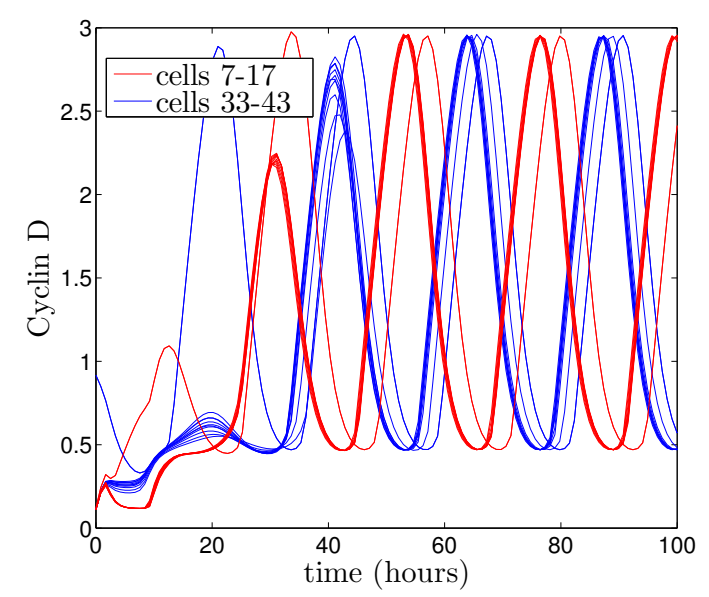

Figure 10: Simulation results illustrating the emergence of cohorts of synchronised cells in larger cellular systems. A one dimensional 50 cell system is simulated where all cells are initially quiescent except cells 12 and 38 which have an initial phase difference of 12 hours. The ten closest cells (in red) to cell 12 are synchronised with it, whilst the ten closest cells (blue) to cell 38 cycle in synchrony with that cell. 
We note that our results regarding cell cycle synchronisation are largely numerical and that the theory of weakly coupled oscillators (Ermentrout and Kopell, 1984; Kopell and Ermentrout, 1986, 1990; Kuramoto, 2003 ) is often used to obtain analytical results. The application of the theory to our model gave contradictory results. For example for the Cyclin D dependent model variant, for the parameter regime in Figure 2 and where $a_{\mathrm{d} 1}^{\prime}=a_{\mathrm{d} 2}^{\prime}$, the theory of weakly coupled oscillators predicts that the asynchronous limit cycle solution is unstable (data not shown). However, this solution is clearly stable as confirmed by the bifurcation analysis (Figures $2 \mathrm{a}$ and $2 \mathrm{c}$ ) and simulation results (Figure 3e). The most likely explanation for this discrepancy is that the coupling strength we use is strong and hence does not allow for the application of the weakly coupled oscillator theory. Indeed, simulation results where the coupling strength is weakened by reducing the ATP diffusion coefficient $D_{\text {ATP }}$ from $1.26 \times 10^{6} \mu \mathrm{m}^{2} \mathrm{hr}^{-1}$ to below $360 \mu \mathrm{m}^{2} \mathrm{hr}^{-1}$ show that, as predicted by the theory, the asynchronous solution is unstable (data not shown). However, a value for $D_{\text {ATP }}$ of $360 \mu \mathrm{m}^{2} \mathrm{hr}^{-1}$, for which the theory gives accurate predictions, is unphysiological as it is several orders of magnitude below what is physically realistic (de Graaf et al., 2000; Bennett et al., 1995).

Our model incorporates the relatively low dimensional cell cycle model of Obeyesekere et al. (1999). We note that more biophysically realistic, albeit higher dimensional, cell cycle models exist (Novak and Tyson, 2004; Swat et al., 2004; Gérard and Goldbeter, 2011; Pfeuty, 2012). It would be interesting to form a radial glia model incorporating these cell cycle models to confirm that the timing of ATP release plays a crucial role in radial glia synchronisation and this presents an interesting avenue of future work. These models are far more complex than the model of Obeyesekere et al. For example the model of Novak and Tyson (2004) has more than four times as many variables and three times as many parameters and that of Gérard and Goldbeter (2009) has nearly eight times as many variables and more than nine times as many parameters. Consequently the dynamics of these models will be rich and will likely permit asynchronous oscillatory solutions of the type encountered in this work and possibly other types too. Because of this, the transient behaviour of the system once quiescent cells are recruited by cycling cells is likely be to important again. Which ATP release pattern gives rise to synchronous entrainment will depend on the delay before a quiescent cell embarks on the cell cycle once it has receive an ATP signal for the particular cell cycle model considered.

In terms of the biological implications of the results of this work, as synchronised cohorts of radial glia shed neurons in uniform sheets which in turn form the regular layered structure of the neocortex, our results 
raise the intriguing possibility that ATP release and its timing may play a crucial role in the formation of the neocortex. It has been argued that various neurodevelopment disorders may be as a result of irregularities in cortical formation. It is plausible that these irregularities may come about because of a malfunction in the timing of ATP release from radial glia which will ultimately lead to irregularities in neocortical formation. It is conceivable that the impact of ATP release on synchronisation could be tested in vitro. By controlling the point during the cell cycle at which hemichannels form and ATP is released different release patterns could be prescribed and their affect on cell cycle synchronisation within clusters of radial glia investigated. 


\section{Appendices}

\section{A Model equations and parameter values}

Our model contains 13 dynamical variables and 41 parameter values for each cell. The parameter values and their description are given in Tables A.1-A.2. See (Barrack et al., 2014) for their deviation. The variables are the concentrations of active Cyclin D/Cdk4 (D), Cyclin E/Cdk2 $(E)$, unphosphorylated retinoblastoma tumour suppressor protein $(\mathrm{RB}, R)$, phosphorylated $\mathrm{RB}$ bound to the $\mathrm{E} 2 \mathrm{~F}$ transcription factor $\left(R_{\mathrm{S}}\right)$, the cell progression indicator $(\mathrm{CPI}, Q)$, intracellular calcium $\left(\left[\mathrm{Ca}^{2+}\right]\right)$, fraction of bound $\mathrm{P} 2 \mathrm{Y}_{1}$ receptors $(\rho)$, proportion of active G-protein $\left(G^{*}\right)$, concentration of $\mathrm{IP}_{3}\left(\left[\mathrm{IP}_{3}\right]\right)$, intracellular ATP concentration ([ATP $\left.\left.\mathrm{I}\right]\right)$, extracellular ATP concentration $\left(\left[\mathrm{ATP}_{\mathrm{E}}\right]\right)$ and the switch function $T$ which models both switch-like $\mathrm{IP}_{3}$ dependent ATP release and switch-like hemichannel opening. In the model cells are indexed by $j$ if coupled by extracellular ATP diffusion on a one dimensional lattice and by the tuple $j, k$ if cells are arranged on a two dimensional lattice. Below we present the equations for the one dimensional model.

\section{Cell cycle dynamics}

$$
\begin{aligned}
\frac{d D_{j}}{d t} & =a_{\mathrm{d} j} \cdot G F_{j}-d_{D j} E_{j} D_{j}, \\
\frac{d E_{j}}{d t} & =a_{\mathrm{E} j}\left(1+a_{\mathrm{f} j}\left(E 2 F_{\mathrm{T} j}-R_{\mathrm{s} j}\right)\right)-d_{\mathrm{E} j} Q_{j} E_{j}, \\
\frac{d R_{j}}{d t} & =\frac{p_{\mathrm{Q} j}\left(R_{\mathrm{T} j}-R_{\mathrm{s} j}-R_{j}\right) Q_{j}}{q_{\mathrm{Q} j}+\left(R_{\mathrm{T} j}-R_{\mathrm{s} j}-R_{j}\right)+Q_{j}}-p_{\mathrm{s} j}\left(E 2 F_{\mathrm{T} j}-R_{\mathrm{s} j}\right) R_{j}, \\
\frac{d R_{\mathrm{s} j}}{d t} & =p_{\mathrm{s} j}\left(E 2 F_{\mathrm{T} j}-R_{\mathrm{s} j}\right) R_{j}-\frac{p_{\mathrm{D} j} R_{\mathrm{s} j} D_{j}}{q_{\mathrm{D} j}+R_{\mathrm{s} j}+D_{j}}-\frac{p_{\mathrm{E} j} R_{\mathrm{s} j} E_{j}}{q_{\mathrm{E} j}+R_{\mathrm{s} j}+E_{j}}, \\
\frac{d Q_{j}}{d t} & =a_{\mathrm{Q} j} E_{j}+\beta\left(E 2 F_{\mathrm{T} j}-R_{\mathrm{s} j}\right)+g_{j} Q_{j}^{2} E_{j}-d_{\mathrm{Q} j} Q_{j}, \\
a_{\mathrm{d} j}\left(\left[\mathrm{Ca}^{2+}\right]_{j}\right) & =a_{\mathrm{d} j}^{\prime}+\gamma_{j}\left(\left[\mathrm{Ca}^{2+}\right]_{j}-\left[\mathrm{Ca}^{2+}\right]_{\mathrm{b} j}\right),
\end{aligned}
$$




\section{ATP mediated calcium release}

$$
\begin{aligned}
\rho_{j} & =\frac{\left[\mathrm{ATP}_{\mathrm{E}}\right]_{j}}{K_{\mathrm{R} j}+\left[\mathrm{ATP}_{\mathrm{E}}\right]_{j}}, \\
G_{j}^{*} & =\frac{\rho_{j}+\nu_{j}}{K_{\mathrm{G} j}+\rho_{j}+\nu_{j}}, \quad \frac{d\left[\mathrm{IP}_{3}\right]_{j}}{d t}=r_{\mathrm{h} j}^{*} G_{j}^{*}-k_{\mathrm{deg} j}\left[\mathrm{IP}_{3}\right]_{j}, \\
\frac{d\left[\mathrm{ATP}_{\mathrm{I}}\right]_{j}}{d t} & =A T P_{\operatorname{prod} j}-A T P_{\mathrm{rel} j}, \quad \frac{d\left[\mathrm{ATP}_{\mathrm{E}}\right]_{j}}{d t}=A T P_{\mathrm{rel} j}+A T P_{\mathrm{dif} j}-A T P_{\mathrm{deg} j}, \\
A T P_{\mathrm{prod} j} & =\alpha_{j}\left(\left[\mathrm{ATP}_{\mathrm{I}}\right]_{\max j}-\left[\mathrm{ATP}_{\mathrm{I}}\right]_{j}\right), \quad A T P_{\operatorname{deg} j}=V_{\mathrm{deg} j} \frac{\left[\mathrm{ATP}_{\mathrm{E}}\right]_{j}}{K_{\operatorname{deg} j}+\left[\mathrm{ATP}_{\mathrm{E}}\right]_{j}}, \\
A T P_{\mathrm{dif} j} & =D_{\mathrm{ATP}} \frac{\left[\mathrm{ATP}_{\mathrm{E}}\right]_{j-1}-2\left[\mathrm{ATP}_{\mathrm{E}}\right]_{j}+\left[\mathrm{ATP}_{\mathrm{E}}\right]_{j+1}}{\Delta x^{2}}, \\
A T P_{\mathrm{rel} j} & =\left(\frac{\left[\mathrm{IP}_{3}\right]_{j}-\left[\mathrm{IP}_{3}\right]_{\min j}}{K_{\mathrm{rel} j}+\left[\mathrm{IP}_{3}\right]_{j}}\right) V_{\mathrm{ATP} j}\left(\left[\mathrm{ATP}_{\mathrm{I}}\right]_{j}-\left[\mathrm{ATP}_{\mathrm{E}}\right]_{j}\right) T_{j}\left(i_{1}-i_{2}\right) T_{j}\left(\left[\mathrm{IP}_{3}\right]-\left[\mathrm{IP}_{3 j}\right]_{\min j}\right), \\
T_{j}(y) & =\frac{1}{2}\left(\tanh \left(\frac{y}{\epsilon}\right)+1\right), \quad\left[\mathrm{Ca}^{2+}\right]_{j}=\left[\mathrm{Ca}^{2+}\right]_{\mathrm{b}}+\frac{p_{1 j}\left[\mathrm{IP}_{3}\right]_{j}^{m j}}{p_{2 j}^{m j}+\left[\mathrm{IP}_{3}\right]_{j}^{m j}}+\frac{p_{3 j}\left[\mathrm{IP}_{3}\right]_{j}^{n j}}{p_{4 j}^{n j}+\left[\mathrm{IP}_{3}\right]_{j}^{n j}} .
\end{aligned}
$$

In two dimensions, all equations are identical except that we replace the subscript $j$ with $j, k$. In addition ATP diffusion (equation (A.11)) is given by

$$
A T P_{\text {dif } j, k}=D_{\mathrm{ATP}} \frac{\left[\mathrm{ATP}_{\mathrm{E}}\right]_{j-1, k}+\left[\mathrm{ATP}_{\mathrm{E}}\right]_{j, k-1}-4\left[\mathrm{ATP}_{\mathrm{E}}\right]_{j, k}+\left[\mathrm{ATP}_{\mathrm{E}}\right]_{j+1, k}+\left[\mathrm{ATP}_{\mathrm{E}}\right]_{j, k+1}}{\Delta x^{2}} .
$$

The cell cycle component given by equations (A.1)-(A.6) is based on the model of Obeyesekere et al. (1999, 1997) for the mammalian cell cycle. Although the cell cycle period will depend on parameter values, for the baseline parameter values given in Tables A.1 and A.2, the period is 27.29 hours for the Cyclin D dependent ATP release variant and 26.85 hours for $R_{\mathrm{S}}$ ATP release model variant. This is consistent with the radial glial cell cycle period of primates (22-55 hours (Kornack and Rakic, 1998)) and close to that of mice (8-18 hours (Takahashi et al., 1995)).

In equation (A.11), $D_{\mathrm{ATP}}$ is the diffusion coefficient and $\Delta x$ is the physical distance between adjacent cells. In one dimension the $\left[\mathrm{ATP}_{\mathrm{E}}\right]_{j-1}-2\left[\mathrm{ATP}_{\mathrm{E}}\right]_{j}+\left[\mathrm{ATP}_{\mathrm{E}}\right]_{j+1}$ term (where $j$ is the cell number) ensures that each cell $j$ is diffusively coupled to its nearest neighbours $j-1$ and $j+1$. When cells are cycling, this allows for the passing of an ATP signal from a cell to its neighbours which then release their own ATP signal. For the Cyclin D dependent ATP release model variant (where hemichannel opening and ATP release occurs when a cell is in mid $\mathrm{G}_{1}$ phase) $\left(i_{1}, i_{2}\right)=\left(D, D_{\mathrm{c}}\right)$ and for the $R_{\mathrm{s}}$ dependent ATP release model variant (where 
hemichannel opening and ATP release occurs when a cell at the $\mathrm{G}_{1} / \mathrm{S}$ transition $)\left(i_{1}, i_{2}\right)=\left(R_{\mathrm{Sc}}, R_{\mathrm{S}}\right)$. 
Table A.1: Parameter values for cell cycle component

\begin{tabular}{|c|c|c|c|}
\hline Index & Parameter & Value & Description \\
\hline 1 & $G F$ & 0.25384 & Growth factor activity \\
\hline 2 & $d_{\mathrm{D}}$ & $0.4 \mathrm{hr}^{-1}$ & $\begin{array}{l}\text { Rate parameter at with which Cyclin D } \\
\text { is degraded by active Cyclin E/Cdk2 }\end{array}$ \\
\hline 3 & $a_{\mathrm{E}}$ & $0.16 \mathrm{hr}^{-1}$ & $\begin{array}{l}\text { E2F independent Cyclin E synthesis rate } \\
\text { parameter }\end{array}$ \\
\hline 4 & $a_{\mathrm{f}}$ & 0.9 & $\begin{array}{l}\text { E2F dependent Cyclin E synthesis rate } \\
\text { parameter }\end{array}$ \\
\hline 5 & $E 2 F_{\mathrm{T}}$ & 1.5 & Total E2F concentration \\
\hline 6 & $d_{\mathrm{E}}$ & $0.2 \mathrm{hr}^{-1}$ & $\begin{array}{l}\text { Rate parameter at with Cyclin E is degraded } \\
\text { by the CPI }\end{array}$ \\
\hline 7 & $p_{\mathrm{Q}}$ & $0.48 \mathrm{hr}^{-1}$ & $\begin{array}{l}\text { Rate parameter at with which the CPI } \\
\text { dephosphorylates RB }\end{array}$ \\
\hline 8 & $q_{\mathrm{Q}}$ & $0.8 \mathrm{hr}^{-1}$ & Michaelis constant \\
\hline 9 & $R_{\mathrm{T}}$ & 2.5 & Total RB \\
\hline 10 & $p_{\mathrm{s}}$ & $0.6 \mathrm{hr}^{-1}$ & $\begin{array}{l}\text { Constant at which free unphosphorylated RB } \\
\text { sequesters E2F }\end{array}$ \\
\hline 11 & $p_{\mathrm{D}}$ & $0.48 \mathrm{hr}^{-1}$ & $\begin{array}{l}\text { Rate parameter at which active } \\
\text { Cyclin D/Cdk4 phosphorylates RB }\end{array}$ \\
\hline 12 & $q_{\mathrm{D}}$ & $0.6 \mathrm{hr}^{-1}$ & Michaelis constant \\
\hline 13 & $p_{\mathrm{E}}$ & $0.096 \mathrm{hr}^{-1}$ & $\begin{array}{l}\text { Rate parameter at which Cyclin E } \\
\text { phosphorylates RB }\end{array}$ \\
\hline 14 & $q_{\mathrm{E}}$ & $0.6 \mathrm{hr}^{-1}$ & Michaelis constant \\
\hline 15 & $a_{\mathrm{Q}}$ & $0.08 \mathrm{hr}^{-1}$ & $\begin{array}{l}\text { Rate parameter of Cyclin E/Cdk2 dependent CPI } \\
\text { production }\end{array}$ \\
\hline 16 & $\beta$ & $0.2 \mathrm{hr}^{-1}$ & $\begin{array}{l}\text { Rate parameter of free } \mathrm{E} 2 \mathrm{~F} \text { dependent } \mathrm{CPI} \\
\text { production }\end{array}$ \\
\hline 17 & $g$ & $0.528 \mathrm{hr}^{-1}$ & CPI autocatalytic reaction rate parameter \\
\hline 18 & $d_{\mathrm{Q}}$ & $1.04 \mathrm{hr}^{-1}$ & CPI degradation rate constant \\
\hline 19 & $a_{\mathrm{d}}^{\prime}$ & $0.41 \mathrm{hr}^{-1}$ & Cyclin D synthesis parameter \\
\hline
\end{tabular}


Table A.2: Parameter values for ATP mediated calcium release component

\begin{tabular}{|c|c|c|c|}
\hline Index & Parameter & Value & Description \\
\hline 20 & $K_{\mathrm{R}}$ & $25 \mu \mathrm{M}$ & $\begin{array}{l}\text { Effective dissociation constant for } \\
\mathrm{P}_{2} \mathrm{Y}_{1} \text { receptor ATP binding }\end{array}$ \\
\hline 21 & $\nu$ & 0.12 & $\begin{array}{l}\text { background G-protein } \\
\text { activation constant }\end{array}$ \\
\hline 22 & $K_{\mathrm{G}}$ & 8.82353 & G-protein dissociation constant \\
\hline 23 & $r_{\mathrm{h}}^{*}$ & $2160 \mu \mathrm{Mhr}^{-1}$ & $\mathrm{IP}_{3}$ production rate \\
\hline 24 & $k_{\mathrm{deg}}$ & $225 \mathrm{hr}^{-1}$ & $\mathrm{IP}_{3}$ degradation rate \\
\hline 25 & $V_{\mathrm{deg}}$ & $7200 \mu \mathrm{Mhr}^{-1}$ & $\begin{array}{l}\text { Michaelis constant } \\
\text { (expected range } 36-7200 \mu \mathrm{Mhr}^{-1} \text { ), }\end{array}$ \\
\hline 26 & $K_{\operatorname{deg}}$ & $50 \mu \mathrm{M}$ & Michaelis constant \\
\hline 27 & $V_{\mathrm{ATP}}$ & $180000 \mathrm{hr}^{-1}$ & ATP release rate constant \\
\hline 28 & {$\left[\mathrm{IP}_{3}\right]_{\min }$} & $0.013 \mu \mathrm{M}$ & $\begin{array}{l}\text { Minimum } \mathrm{IP}_{3} \text { concentration } \\
\text { for ATP release }\end{array}$ \\
\hline 29 & $K_{\text {rel }}$ & $10 \mu \mathrm{M}$ & Kinetic parameter \\
\hline 30 & $\gamma$ & $2 \mu \mathrm{M}^{-1} \mathrm{hr}^{-1}$ & Calcium coupling strength \\
\hline 31 & {$\left[\mathrm{Ca}^{2+}\right]_{b}$} & $0.0159835 \mu \mathrm{M}$ & Steady state calcium concentration \\
\hline 32 & $\alpha$ & $298.8 \mathrm{hr}^{-1}$ & ATP production rate constant \\
\hline 33 & {$\left[\mathrm{ATP}_{\mathrm{I}}\right]_{\max }$} & $500 \mu \mathrm{M}$ & Maximum internal ATP concentration \\
\hline 34 & $\epsilon$ & $0.01 \mu \mathrm{M}^{-1}$ & Stiffness of switch function \\
\hline 35 & $p_{1}$ & 0.514987 & Hill function coefficient \\
\hline 36 & $p_{2}$ & 1.31319 & Hill function coefficient \\
\hline 37 & $p_{3}$ & 0.332195 & Hill function coefficient \\
\hline 38 & $p_{4}$ & 0.787902 & Hill function coefficient \\
\hline 39 & $m$ & 24.1946 & Hill function coefficient \\
\hline 40 & $n$ & 9.79183 & Hill function coefficient \\
\hline 41 & $D_{\mathrm{c}}$ & 0.5 & Critical Cyclin D concentration above which ATP \\
\hline 42 & $R_{\mathrm{sc}}$ & 1 & Critical $R_{\mathrm{S}}$ concentration below which ATP \\
\hline 43 & $D_{\text {ATP }}$ & $1260000 \mu \mathrm{m}^{2} \mathrm{hr}^{-1}$ & ATP diffusion coefficient \\
\hline 44 & $\Delta x$ & $10 \mu \mathrm{m}$ & distance between adjacent cells \\
\hline
\end{tabular}




\section{References}

D.S. Barrack, R. Thul, and M.R. Owen. Modelling the coupling between intracellular calcium release and the cell cycle during cortical brain development. J. Theor. Biol., 347:17-32, 2014.

S.A. Bayer, J. Altman, R.J. Russo, and Xin Zhang. Timetables of neurogenesis in the human brain based on experimentally determined patterns in the rat. Neurotoxicology, 14(1):83-144, 1993.

M.R. Bennett, L. Farnell, W.G. Gibson, and S. Karunanithi. Quantal transmission at purinergic junctions: stochastic interaction between ATP and its receptors. Biophys. J., 68(3):925-935, 1995.

M.R. Bennett, L. Farnell, and W.G. Gibson. A quantitative model of purinergic junctional transmission of calcium waves in astrocyte networks. Biophys. J., 89(4):2235-2250, 2005.

J.M. Berridge. Calcium signalling and cell proliferation. BioEssays, 17(6):491-500, 1995.

M.J. Berridge, P. Lipp, and M.D. Bootman. The versatility and universality of calcium signalling. Nat. Rev. Mol. Cell Biol., 1(1):11-21, 2000.

K.S. Bittman and J.J. LoTurco. Differential regulation of connexin 26 and 43 in murine neocortical precursors. Cereb. Cortex, 9(2):188-195, 1999.

G. Buzsáki and A. Draguhn. Neuronal oscillations in cortical networks. Science, 304(5679):1926-1929, 2004.

V.S. Caviness, T. Takahashi, and R.S. Nowakowski. Numbers, time and neocortical neuronogenesis: a general developmental and evolutionary model. Trends Neurosci, 18(9):379-383, 1995.

P. Coti Bertrand, J.R. O'Kusky, and S.M. Innis. Maternal dietary (n-3) fatty acid deficiency alters neurogenesis in the embryonic rat brain. J. Nutr., 136(6):1570-1575, 2006.

R.A. de Graaf, A. van Kranenburg, and K. Nicolay. In Vivo ${ }^{31}$ P-NMR Diffusion Spectroscopy of ATP and Phosphocreatine in Rat Skeletal Muscle. Biophys. J., 78(4):1657-1664, 2000.

A. Einstein. Über die von der molekularkinetischen theorie der wärme geforderte bewegung von in ruhenden flüssigkeiten suspendierten teilchen. Ann. Phys, 322(8):549-560, 1905.

G.B. Ermentrout and N. Kopell. Frequency plateaus in a chain of weakly coupled oscillators, i. SIAM J discrete Math, 15(2):215-237, 1984. 
C. Gérard and A. Goldbeter. Temporal self-organization of the cyclin/Cdk network driving the mammalian cell cycle. Proc. Natl. Acad. Sci. U.S.A., 106(51):21643-21648, 2009.

C. Gérard and A. Goldbeter. A skeleton model for the network of cyclin-dependent kinases driving the mammalian cell cycle. Interface Focus, 1(1):24-35, 2011.

C.R. Kahl and A.R. Means. Regulation of cell cycle progression by calcium/calmodulin-dependent pathways. Endocr. Rev., 24(6):719-736, 2003.

C.R. Kahl and A.R. Means. Regulation of Cyclin D1/Cdk 4 complexes by calcium/calmodulin-dependent protein kinase I. J. Biol. Chem., 279(15):15411-15419, 2004.

H. Kitajima, Y. Katsuta, and H. Kawakami. Bifurcations of periodic solutions in a coupled oscillator with voltage ports. IEICE Trans. Fundamentals, 81(3):476-482, 1998.

N. Kopell and G.B. Ermentrout. Symmetry and phaselocking in chains of weakly coupled oscillators. Commun Pur Appl Math, 39(5):623-660, 1986.

N. Kopell and G.B. Ermentrout. Phase transitions and other phenomena in chains of coupled oscillators. SIAM J Appl Math, 50(4):1014-1052, 1990.

D. R. Kornack and Pasko Rakic. Changes in cell-cycle kinetics during the development and evolution of primate neocortex. Proc. Natl. Acad. Sci. U.S.A., 95(3):1242-1246, 1998.

Y. Kuramoto. Chemical oscillations, waves, and turbulence. Courier Dover Publications. 2003.

I. Kuznetsov. Elements of applied bifurcation theory. Springer. 1998.

H. Li, T.F. Liu, A. Lazrak, C. Peracchia, G.S. Goldberg, P.D. Lampe, and R.G. Johnson. Properties and regulation of gap junctional hemichannels in the plasma membranes of cultured cells. J. Cell Biol., 134(4): 1019-1030, 1996.

T.A. Morris, R.J. DeLorenzo, and R.M. Tombes. CaMKII inhibition reduces Cyclin D1 levels and enhances the association of $\mathrm{p} 27^{\mathrm{Kip} 1}$ with Cdk2 to cause G1 arrest in NIH 3T3 cells. Exp. Cell Res., 240(2):218-227, 1998.

S.C. Noctor, A.C. Flint, T.A. Weissman, R.S. Dammerman, and A.R. Kriegstein. Neurons derived from radial glial cells establish radial units in neocortex. Nature, 409(6821):714-720, 2001. 
B. Novak and J.J. Tyson. A model for restriction point control of the mammalian cell cycle. J. Theor. Biol., 230(4):563-579, 2004.

M.N. Obeyesekere, E.S. Knudsen, J.Y.J. Wang, and S.O. Zimmerman. A mathematical model of the regulation of the G1 phase of Rb+/+ and Rb-/-mouse embryonic fibroblasts and an osteosarcoma cell line. Cell Prolif., 30(3-4):171-194, 1997.

M.N. Obeyesekere, S.O. Zimmerman, E.S. Tecarro, and G. Auchmuty. A model of cell cycle behavior dominated by kinetics of a pathway stimulated by growth factors. Bull. Math. Biol., 61(5):917-934, 1999.

T. Oki, K. Nishimura, J. Kitaura, K. Togami, A. Maehara, K. Izawa, A. Sakaue-Sawano, A. Niida, S. Miyano, and H. Aburatani. A novel cell-cycle-indicator, mVenus-p27K-, identifies quiescent cells and visualizes G0-G1 transition. Sci. Rep., 4, 2014.

B. Pfeuty. Strategic cell-cycle regulatory features that provide mammalian cells with tunable G1 length and reversible G1 arrest. PLoS ONE, 7(4):e35291, 2012.

D. Postnov, S.K. Han, and H. Kook. Synchronization of diffusively coupled oscillators near the homoclinic bifurcation. Phys. Rev. E, 60(3):2799-2807, 1999.

G. Rasmussen and C. Rasmussen. Calmodulin-dependent protein kinase II is required for G1/S progression in HeLa cells. Biochem Cell Biol., 73(3-4):201-207, 1995.

A.D. Short, J. Bian, T.K. Ghosh, R.T. Waldron, S.L. Rybak, and D.L. Gill. Intracellular ca2+ pool content is linked to control of cell growth. Proc. Natl. Acad. Sci. U.S.A., 90(11):4986-4990, 1993.

B. Silverman. Density estimation for statistics and data analysis. Chapman \& Hall. 1986.

M. Swat, A. Kel, and H. Herzel. Bifurcation analysis of the regulatory modules of the mammalian G1/S transition. Bioinformatics, 20(10):1506-1511, 2004.

T Takahashi, R. S. Nowakowski, and V. S. Caviness. The cell cycle of the pseudostratified ventricular epithelium of the embryonic murine cerebral wall. J. Neurosci., 15(9):6046-6057, 1995.

R.M. Tombes, S. Grant, E.H. Westin, and G. Krystal. G1 cell cycle arrest and apoptosis are induced in NIH $3 \mathrm{~T} 3$ cells by KN-93, an inhibitor of CaMK-II (the multifunctional $\mathrm{Ca}^{2+} / \mathrm{CaM}$ kinase). Cell Growth Differ., 6 (9):1063-1070, 1995. 
D. Wang. Emergent synchrony in locally coupled neural oscillators. IEEE Trans. Neural Netw., 6(4):941-948, 1995.

T.A. Weissman, P.A. Riquelme, L. Ivic, A.C. Flint, and A.R. Kriegstein. Calcium waves propagate through radial glial cells and modulate proliferation in the developing neocortex. Neuron, 43(5):647-661, 2004. 